\title{
Dynamics of a reaction-diffusion SIRS model with general incidence rate in a heterogeneous environment
}

\author{
Eric Avila-Vales(1) and Ángel G. C. Pérez(D)
}

\begin{abstract}
In this paper, we study a diffusive SIRS-type epidemic model with transfer from the infectious to the susceptible class. Our model includes a general nonlinear incidence rate and spatially heterogeneous diffusion coefficients. We compute the basic reproduction number $\mathcal{R}_{0}$ of our model and establish the global stability of the disease-free steady state when $\mathcal{R}_{0}<1$. Furthermore, we study the uniform persistence when $\mathcal{R}_{0}>1$ and perform a bifurcation analysis for a special case of our model. Some numerical simulations are presented to illustrate the dynamics of the solutions as the model parameters are varied.
\end{abstract}

Mathematics Subject Classification. 35K57, 92D30.

Keywords. Reaction-diffusion, Global stability, Uniform persistence, Bifurcation analysis.

\section{Introduction}

The spread of infectious diseases within human populations is an important topic of research in mathematical modelling that has become widely studied in recent times. Many different models, such as the SI, SIS, SIR and SIRS types, have been proposed to better understand the underlying transmission mechanism of infections. In these models, the population under study is divided into several classes or subpopulations, such as susceptible $(\mathrm{S})$, infected $(\mathrm{I})$ and recovered $(\mathrm{R})$.

The incidence rate of a disease, which measures how many people become infected by that disease per time unit, plays an important role in the dynamics of epidemic models. Traditionally, the incidence rate has been assumed to be bilinear with respect to the number of susceptible individuals $(S)$ and the number of infected individuals $(I)$. However, many other functions have been proposed to model the transmission of infectious diseases. In general, the incidence function can be written in the form $F(S, I)$, where the function $F$ satisfies some common properties, such as the following:

(A1') $F(S, I)=I F_{1}(S, I)$ with $F, F_{1} \in C^{1}\left(\mathbb{R}_{+}^{2}\right)$.

(A2') $F(0, I)=F(S, 0)=0$ for all $S, I \geq 0$.

(A3') $\frac{\partial F}{\partial I}(S, 0)>0$ for all $S>0 ; \frac{\partial F_{1}}{\partial S}(S, I)>0$ and $\frac{\partial F_{1}}{\partial I}(S, I) \leq 0$ for all $S, I \geq 0$.

(A4') There exists a positive constant $\beta$ such that $F(S, I) \leq \beta S I$ for all $S, I \geq 0$.

Many specific forms of incidence functions commonly studied in the literature satisfy (A1')-(A4'). Some examples are:

- $F(S, I)=\beta S I$ (bilinear) $[1]$;

- $F(S, I)=\beta S I /\left(1+a_{1} I\right)$ (saturated with respect to infectives) [2];

- $F(S, I)=\beta S I /\left(1+a_{2} S\right)$ (saturated with respect to susceptibles) [3];

- $F(S, I)=\beta S I /\left(1+k_{1} I+k_{2} S\right)$ (Beddington-DeAngelis) [4];

- $F(S, I)=\beta S I /\left[\left(1+k_{1} I\right)\left(1+k_{2} S\right)\right]$ (Crowley-Martin) [5]; 
It should be noted that the above forms of incidence rate are monotone with respect to $S, I$ and concave with respect to $I$. On the other hand, non-monotone incidence rates have been used to describe the effect of media coverage: at the beginning of an epidemic, the population has little awareness of the preventive measures, so the contact rate increases rapidly. As the population becomes more aware of risks, they take measures to control the outbreak, so the number of infectious contacts decreases. Some examples of non-monotone incidence functions are the following:

- $F(S, I)=\beta e^{-m I} S I$ with $m>0[6]$;

- $F(S, I)=\beta S I /\left(1+a S+b I^{2}\right)$ with $a, b>0[7]$

- $F(S, I)=\beta S I /\left(1+\omega_{1} I+\omega_{2} I^{2}\right)$ with $\omega_{1}, \omega_{2}>0[8]$

Given the wide variety of forms that have been proposed for the incidence rate, some authors have opted to study epidemic models that include a general class of incidence functions. This has the advantage that the results proved for particular models can be generalized to a broader class of models, and the researchers can focus on other features, such as spatial heterogeneity that could yield more complicated dynamics or bifurcations.

A SIRS model with transfer from the infected to the susceptible class was studied in [9] using the system of ordinary differential equations

$$
\begin{aligned}
\frac{\mathrm{d} S}{\mathrm{~d} t} & =\Lambda-\mu S-S f(I)+\gamma_{1} I+\delta R, \\
\frac{\mathrm{d} I}{\mathrm{~d} t} & =S f(I)-\left(\mu+\alpha+\gamma_{1}+\gamma_{2}\right) I, \\
\frac{\mathrm{d} R}{\mathrm{~d} t} & =\gamma_{2} I-\mu R-\delta R,
\end{aligned}
$$

where $S(t), I(t)$ and $R(t)$ denote the number of susceptible, infected, and recovered individuals at time $t$, respectively, and the parameters are interpreted as follows:

- $\Lambda$ : recruitment rate of susceptible population.

- $\mu$ : natural death rate.

- $\alpha$ : disease-induced death rate.

- $\gamma_{1}$ : transfer rate from the infected class to the susceptible class.

- $\gamma_{2}$ : transfer rate from the infected class to the recovered class.

- $\delta$ : rate of immunity loss.

The authors in [9] considered an incidence function of the form $S f(I)$ and proved that the threshold dynamics for model (1.1) is completely determined by the basic reproduction number, which is denoted as $\mathcal{R}_{0}$. Variants of such model were later studied in [10] and [11] using more general incidence rates, which take the form $f(S, I)$. The authors in $[10,11]$ showed that the generalized model retains its threshold dynamics under certain conditions of the parameters.

On the other hand, we should consider that the individuals of the population under study may move randomly in the space. Several studies on modelling of infectious diseases such as influenza [12], cholera [13], dengue [14], brucellosis [15] and COVID-19 [16] have highlighted the importance of individual motility in the dynamics of an outbreak. Thus, it is appropriate to consider epidemic models based on reactiondiffusion equations, where the moving patterns for susceptible, infected and recovered individuals are modelled through the diffusion rate of each subpopulation.

Moreover, many communicable diseases occur in a heterogeneous environment due to the differences in environmental conditions such as humidity, temperature or the varying availability of medical resources. This has led some researchers to study epidemic models where some of the parameters depend on a spatial variable $x$. In particular, the transmission rate of the disease may be represented by a function that depends not only on the number of susceptible and infected individuals, but also on the spatial location. For example, a SIRS-type model with diffusion and heterogeneous parameters was studied in [12] with the incidence function $f(x, I) S$. Other reaction-diffusion models have been proposed in [17] 
using the general incidence function $\beta(x) f(S, I)$ and in [13] using the function $f(x, S, I)$, which take into account both the dependence on the spatial variable and the non-linearity with respect to $S$ and $I$.

A diffusive epidemic model based on system (1.1) was studied by Yang et al. in [18], where the authors established the global attractiveness of the disease-free equilibrium when $\mathcal{R}_{0}<1$ and the persistence of the disease when $\mathcal{R}_{0}>1$. Although the authors in [18] included a general incidence rate of the form $\beta(x) f(S, I)$ and spatially variable parameters, they assumed that the diffusion coefficients for the susceptible, infected and recovered individuals are all equal to a constant $D$. In reality, there could be differences in the motility patterns of these subpopulations due to the individuals changing their behaviour when they get the disease, and the diffusion rate may also vary with the spatial location.

Based on the above discussion, we propose here a diffusive version of the SIRS model (1.1) that includes three different diffusion coefficients with spatial heterogeneity. The model to be studied is given by the following system of partial differential equations:

$$
\begin{array}{ll}
\partial_{t} S=\nabla \cdot\left(d_{S}(x) \nabla S\right)+\Lambda(x)-\mu(x) S-F(x, S, I)+\gamma_{1}(x) I+\delta(x) R, & x \in \Omega, t>0, \\
\partial_{t} I=\nabla \cdot\left(d_{I}(x) \nabla I\right)+F(x, S, I)-\left(\mu+\alpha+\gamma_{1}+\gamma_{2}\right)(x) I, & x \in \Omega, t>0, \\
\partial_{t} R=\nabla \cdot\left(d_{R}(x) \nabla R\right)+\gamma_{2}(x) I-\mu(x) R-\delta(x) R, & x \in \Omega, t>0, \\
{\left[d_{S}(x) \nabla S(x, t)\right] \cdot \mathbf{n}=\left[d_{I}(x) \nabla I(x, t)\right] \cdot \mathbf{n}=\left[d_{R}(x) \nabla R(x, t)\right] \cdot \mathbf{n}=0,} & x \in \partial \Omega, t>0,
\end{array}
$$

subject to the initial conditions

$$
S(x, 0)=S_{0}(x) \geq 0, \quad I(x, 0)=I_{0}(x) \geq 0, \quad R(x, 0)=R_{0}(x) \geq 0, \quad x \in \Omega .
$$

Here, the variables $S(x, t), I(x, t)$ and $R(x, t)$ represent the number of susceptible, infected, and recovered individuals, respectively, at position $x$ and time $t$, while $\nabla$ is the gradient operator. We assume that the domain $\Omega$ is a connected, bounded subset of $\mathbb{R}^{n}$ with smooth boundary $\partial \Omega$. The positive functions $d_{S}(\cdot), d_{I}(\cdot)$ and $d_{R}(\cdot)$ denote the spatially heterogeneous diffusion coefficients for each subpopulation. The spatially dependent parameters $\Lambda(\cdot), \mu(\cdot), \alpha(\cdot), \gamma_{1}(\cdot), \gamma_{2}(\cdot)$ and $\delta(\cdot)$ have the same meaning as for model (1.1); for biological reasons, they are assumed to be strictly positive (except $\gamma_{1}$, which is nonnegative) and uniformly bounded on $\bar{\Omega}$. Furthermore, for the model to be well-posed, we assume that

$$
\Lambda(\cdot), \mu(\cdot), \alpha(\cdot), \gamma_{1}(\cdot), \gamma_{2}(\cdot), \delta(\cdot) \in C^{0}(\bar{\Omega}, \mathbb{R}) .
$$

Based on (A1')-(A4'), we make the following assumptions on the incidence function $F(x, S, I)$ for model (1.2):

(A1) $F(x, S, I)=I F_{1}(x, S, I)$ with $F, F_{1} \in C^{1}\left(\Omega \times \mathbb{R}_{+}^{2}\right)$.

(A2) $F(x, 0, I)=F(x, S, 0)=0$ for all $x \in \Omega, S, I \geq 0$.

(A3) $\frac{\partial F}{\partial I}(x, S, 0)>0$ for all $x \in \Omega, S>0 ; \frac{\partial F_{1}}{\partial S}(x, S, I)>0$ and $\frac{\partial F_{1}}{\partial I}(x, S, I) \leq 0$ for all $x \in \Omega, S, I \geq 0$.

(A4) There exists a Hölder continuous function $\beta: \Omega \rightarrow \mathbb{R}_{+}$such that $F(x, S, I) \leq \beta(x) S I$ for all $x \in \Omega$, $S, I \geq 0$.

The rest of this paper is organized as follows. First, in Sect. 2, we prove the existence of bounded global solutions for our model. Next, in Sect. 3, we compute the basic reproduction number and study the stability of the disease-free steady state. In Sect. 4, we study the persistence of the model and the existence of endemic equilibria. In Sect. 5, we perform a bifurcation analysis for a special case of our model. In Sect. 6, we carry out some numerical simulations. Finally, we provide some concluding remarks in Sect. 7.

\section{Basic properties of the model}

Let $\mathbb{X}:=C\left(\bar{\Omega}, \mathbb{R}^{3}\right)$ be the Banach space with the supremum norm $\|\cdot\|$, and define $\mathbb{X}^{+}:=C\left(\bar{\Omega}, \mathbb{R}_{+}^{3}\right)$. Then, $\left(\mathbb{X}, \mathbb{X}^{+}\right)$is a strongly ordered space. 
We will now prove the existence of unique global solutions for our model. For that, we introduce the notation

$$
\psi^{-}:=\min _{x \in \bar{\Omega}} \psi(x), \quad \psi^{+}:=\max _{x \in \bar{\Omega}} \psi(x),
$$

where $\psi$ is any of $\Lambda, \mu, \alpha, \gamma_{1}, \gamma_{2}, \delta$ or $\beta$.

Theorem 2.1. For every initial value function $\phi:=\left(\phi_{1}, \phi_{2}, \phi_{3}\right) \in \mathbb{X}^{+}$, model (1.2) has a unique solution $U(\cdot, t ; \phi)=(S(\cdot, t ; \phi), I(\cdot, t ; \phi), R(\cdot, t ; \phi))$ with $U(\cdot, 0 ; \phi)=\phi$ defined on $[0, \infty)$.

Proof. Define $d_{1}(x)=d_{S}(x), d_{2}(x)=d_{I}(x), d_{3}(x)=d_{R}(x), \pi_{1}(x)=\mu(x), \pi_{2}(x)=\mu(x)+\alpha(x)+\gamma_{1}(x)+$ $\gamma_{2}(x)$ and $\pi_{3}(x)=\mu(x)+\delta(x)$. For $i=1,2,3$, let $\Gamma_{i}(t): C(\bar{\Omega}, \mathbb{R}) \rightarrow C(\bar{\Omega}, \mathbb{R})$ be the $C_{0}$ semigroup associated with $\nabla \cdot\left(d_{i}(\cdot) \nabla\right)-\pi_{i}(\cdot)$ subject to the Neumann boundary condition. Then,

$$
\left(\Gamma_{i}(t) \phi\right)(x)=\int_{\Omega} T_{i}(x, y, t) \phi(y) \mathrm{d} y, \quad \forall t>0, \phi \in C(\bar{\Omega}, \mathbb{R}),
$$

where $T_{i}(x, y, t)$ represents the Green function associated with $\nabla \cdot\left(d_{i}(\cdot) \nabla\right)-\pi_{i}(\cdot)$ subject to the Neumann boundary condition. By $\left[19\right.$, Corollary 7.2.3], it follows that $\Gamma(t):=\left(\Gamma_{1}(t), \Gamma_{2}(t), \Gamma_{3}(t)\right)$ is strongly positive and compact for each $t>0$.

Following $[20,21]$, we can see that there exist constants $M_{i}>0(i=1,2,3)$ such that

$$
\left\|\Gamma_{i}(t)\right\| \leq M_{i} e^{\alpha_{i} t} \quad \text { for all } t \geq 0,
$$

where $\alpha_{i}<0$ is the principal eigenvalue of $\nabla \cdot\left(d_{i}(\cdot) \nabla\right)-\pi_{i}(\cdot)$ subject to the Neumann boundary condition. by

For every initial value function $\phi=\left(\phi_{1}(\cdot), \phi_{2}(\cdot), \phi_{3}(\cdot)\right) \in \mathbb{X}^{+}$, we define $G=\left(G_{1}, G_{2}, G_{3}\right): \mathbb{X}^{+} \rightarrow \mathbb{X}$

$$
\begin{aligned}
& G_{1}(\phi)(x)=\Lambda(x)-F\left(x, \phi_{1}(x), \phi_{2}(x)\right)+\gamma_{1}(x) \phi_{2}(x)+\delta(x) \phi_{3}(x), \\
& G_{2}(\phi)(x)=F\left(x, \phi_{1}(x), \phi_{2}(x)\right), \\
& G_{3}(\phi)(x)=\gamma_{2}(x) \phi_{2}(x) .
\end{aligned}
$$

Then, model (1.2) can be rewritten as the integral equation

$$
U(t)=\Gamma(t) \phi+\int_{0}^{t} \Gamma(t-s) G(U(s)) \mathrm{d} s
$$

where $U(t)=(S(t), I(t), R(t))^{T}$. It is easy to see that the subtangential condition in [22, Corollary 4$]$ is satisfied. Thus, the model has a unique positive solution $(S(\cdot, t ; \phi), I(\cdot, t ; \phi), R(\cdot, t ; \phi))$ on $[0, \tau)$, where $0<\tau \leq+\infty$.

We will now prove that the local solution can be extended to a global one, i.e. $\tau=+\infty$.

Suppose by contradiction that $\tau<+\infty$. By the theory of abstract functional differential equations (see [22, Theorem 2]), we know that

$$
\|U(x, t, \phi)\| \rightarrow+\infty \text { as } t \rightarrow \tau .
$$

Hence, it suffices to prove that the solution is bounded on $\Omega \times[0, \tau)$. To this end, we define

$$
K(t)=\int_{\Omega}[S(x, t)+I(x, t)+R(x, t)] \mathrm{d} x .
$$

By the divergence theorem [23, Theorem 3.7] and the homogeneous Neumann boundary conditions, we have

$$
\int_{\Omega} \nabla \cdot\left(d_{S}(x) \nabla S\right) \mathrm{d} x=0, \quad \int_{\Omega} \nabla \cdot\left(d_{I}(x) \nabla I\right) \mathrm{d} x=0, \quad \int_{\Omega} \nabla \cdot\left(d_{R}(x) \nabla R\right) \mathrm{d} x=0 .
$$


Thus,

$$
\begin{aligned}
\frac{\mathrm{d}}{\mathrm{d} t} K(t) & =\int_{\Omega}[\Lambda(x)-\mu(x)(S(x, t)+I(x, t)+R(x, t))-\alpha(x) I(x, t)] \mathrm{d} x \\
& \leq \Lambda^{+}|\Omega|-\mu^{-} K(t), \quad \forall t \in[0, \tau) .
\end{aligned}
$$

By the comparison principle, there exists a constant $N_{1}>0$ and $t_{1}>0$ such that $K(t) \leq N_{1}$ for all $t \in\left[t_{1}, \tau\right)$. Consequently,

$$
\int_{\Omega} S(x, t) \mathrm{d} x \leq N_{1}, \quad \int_{\Omega} I(x, t) \mathrm{d} x \leq N_{1}, \quad \int_{\Omega} R(x, t) \mathrm{d} x \leq N_{1}, \quad \forall t \in\left[t_{1}, \tau\right) .
$$

Next, denote by $\tau_{j}^{i}$ the eigenvalue of $\nabla \cdot\left(d_{i}(\cdot) \nabla\right)-\pi_{i}(\cdot)$ subject to the Neumann boundary condition corresponding to the eigenfunction $\varphi_{j}^{i}(x)$, such that $\tau_{1}^{i}>\tau_{2}^{i} \geq \tau_{3}^{i} \geq \cdots \geq \tau_{j}^{i} \geq \cdots$ for $i=1,2,3$. From [24, Chapter 5], we can write

$$
T_{i}(x, y, t)=\sum_{j \geq 1} \exp \left(\tau_{j}^{i} t\right) \varphi_{j}^{i}(x) \varphi_{j}^{i}(y) .
$$

Since $\varphi_{j}^{i}$ is uniformly bounded, there exists $\omega>0$ such that

$$
T_{i}(x, y, t) \leq \omega \sum_{j \geq 1} \exp \left(\tau_{j}^{i} t\right), \quad \forall t>0, x, y \in \bar{\Omega} .
$$

For $i=1,2,3$, define

$$
d_{i}^{-}:=\min _{x \in \bar{\Omega}} d_{i}(x) \quad \text { and } \quad \pi_{i}^{-}:=\min _{x \in \bar{\Omega}} \pi_{i}(x) .
$$

Denote by $\rho_{j}^{i}$ the eigenvalues of $\nabla \cdot\left(d_{i}^{-} \nabla\right)-\pi_{i}^{-}$subject to the Neumann boundary condition, such that $-\pi_{i}^{-}=\rho_{1}^{i}>\rho_{2}^{i} \geq \rho_{3}^{i} \geq \cdots \geq \rho_{j}^{i} \geq \cdots$. Then, $-\rho_{j}^{i}$ and $-\tau_{j}^{i}$ are the $j$-th eigenvalues of $-\nabla \cdot\left(d_{i}^{-} \nabla\right)+\pi_{i}^{-}$ and $-\nabla \cdot\left(d_{i}(\cdot) \nabla\right)+\pi_{i}(\cdot)$, respectively, subject to the Neumann boundary condition.

For all $x \in \Omega$ and $z \in \mathbb{R}$, we have $d_{i}^{-} z^{2} \leq d_{i}(x) z^{2}$ and $\pi_{i}^{-} \leq \pi_{i}(x)$. Then, by [25, Theorem 2.4.7], we know that $-\rho_{j}^{i} \leq-\tau_{j}^{i}$ for all $j=1,2, \ldots$, which implies that $\rho_{j}^{i} \geq \tau_{j}^{i}$ for all $j=1,2, \ldots$. Since $\rho_{j}^{i}$ decreases like $-j^{2}$, there exists $w_{i}>0$ such that

$$
T_{i}(x, y, t) \leq \omega \sum_{j \geq 1} \exp \left(\rho_{j}^{i} t\right) \leq w_{i} \exp \left(\rho_{1}^{i} t\right)=w_{i} \exp \left(-\pi_{i}^{-} t\right), \quad \forall t>0, x, y \in \bar{\Omega}, i=1,2,3 .
$$

Using (2.2) together with (2.1) and (2.4), we obtain

$$
\begin{aligned}
S(x, t)= & \Gamma_{1}(t) S\left(x, t_{1}\right) \\
& +\int_{t_{1}}^{t} \Gamma_{1}(t-s)\left[\Lambda(x)-F(x, S(x, t), I(x, t))+\gamma_{1}(x) I(x, t)+\delta(x) R(x, t)\right] \mathrm{d} s \\
\leq & \Gamma_{1}(t) S\left(x, t_{1}\right)+\int_{t_{1}}^{t} \Gamma_{1}(t-s)\left[\Lambda(x)+\gamma_{1}(x) I(x, t)+\delta(x) R(x, t)\right] \mathrm{d} s \\
\leq & M_{1} e^{\alpha_{1}\left(t-t_{1}\right)}\left\|S\left(\cdot, t_{1}\right)\right\|+\int_{t_{1}}^{t} \int_{\Omega}^{t} T_{1}(x, y, t-s)\left[\Lambda(x)+\gamma_{1}(x) I(x, t)+\delta(x) R(x, t)\right] \mathrm{d} y \mathrm{~d} s \\
\leq & M_{1} e^{\alpha_{1}\left(t-t_{1}\right)}\left\|S\left(\cdot, t_{1}\right)\right\|+\int_{t_{1}}^{t} w_{1} e^{-(t-s) \pi_{1}^{-}}\left[\Lambda^{+}+\gamma_{1}^{+} \int_{\Omega} I(y, s) \mathrm{d} y+\delta^{+} \int_{\Omega} R(y, s) \mathrm{d} y\right] \mathrm{d} s
\end{aligned}
$$




$$
\begin{aligned}
& \leq M_{1} e^{\alpha_{1}\left(t-t_{1}\right)}\left\|S\left(\cdot, t_{1}\right)\right\|+\int_{t_{1}}^{t} w_{1} e^{-(t-s) \pi_{1}^{-}}\left(\Lambda^{+}+\gamma_{1}^{+} N_{1}+\delta^{+} N_{1}\right) \mathrm{d} s \\
& =M_{1} e^{\alpha_{1}\left(t-t_{1}\right)}\left\|S\left(\cdot, t_{1}\right)\right\|+w_{1}\left(\Lambda^{+}+\gamma_{1}^{+} N_{1}+\delta^{+} N_{1}\right) \frac{1-e^{-\left(t-t_{1}\right) \pi_{1}^{-}}}{\pi_{1}^{-}} \\
& \leq M_{1} e^{-\alpha_{1} t_{1}}\left\|S\left(\cdot, t_{1}\right)\right\|+\frac{w_{1}\left(\Lambda^{+}+\gamma_{1}^{+} N_{1}+\delta^{+} N_{1}\right)}{\mu^{-}}, \quad \forall t \in\left[t_{1}, \tau\right) .
\end{aligned}
$$

Hence,

$$
\|S(\cdot, t)\| \leq M_{1} e^{-\alpha_{1} t_{1}}\left\|S\left(\cdot, t_{1}\right)\right\|+\frac{w_{1}\left(\Lambda^{+}+\gamma_{1}^{+} N_{1}+\delta^{+} N_{1}\right)}{\mu^{-}}=: N_{2}, \quad \forall t \in\left[t_{1}, \tau\right) .
$$

From the second equation of (1.2) and assumption (A4), we get

$$
\begin{aligned}
I(x, t) & =\Gamma_{2}(t) I\left(x, t_{1}\right)+\int_{t_{1}}^{t} \Gamma_{2}(t-s) F(x, S(x, t), I(x, t)) \mathrm{d} s \\
& \leq \Gamma_{2}(t) I\left(x, t_{1}\right)+\int_{t_{1}}^{t} \Gamma_{2}(t-s) \beta(x) S(x, t) I(x, t) \mathrm{d} s \\
& \leq M_{2} e^{\alpha_{2}\left(t-t_{1}\right)}\left\|I\left(\cdot, t_{1}\right)\right\|+\int_{t_{1}}^{t} \int_{\Omega} T_{2}(x, y, t-s) \beta(x) S(x, t) I(x, t) \mathrm{d} y \mathrm{~d} s \\
& \leq M_{2} e^{\alpha_{2}\left(t-t_{1}\right)}\left\|I\left(\cdot, t_{1}\right)\right\|+w_{2} \beta^{+} N_{1} N_{2} \frac{1-e^{-\left(t-t_{1}\right) \pi_{2}^{-}}}{\pi_{2}^{-}} \\
& \leq M_{2} e^{-\alpha_{2} t_{1}}\left\|I\left(\cdot, t_{1}\right)\right\|+\frac{w_{2} \beta^{+} N_{1} N_{2}}{\mu^{-}+\alpha^{-}+\gamma_{1}^{-}+\gamma_{2}^{-}}, \quad \forall t \in\left[t_{1}, \tau\right) .
\end{aligned}
$$

Hence, we obtain

$$
\|I(\cdot, t)\| \leq M_{2} e^{-\alpha_{2} t_{1}}\left\|I\left(\cdot, t_{1}\right)\right\|+\frac{w_{2} \beta^{+} N_{1} N_{2}}{\mu^{-}+\alpha^{-}+\gamma_{1}^{-}+\gamma_{2}^{-}}=: N_{3}, \quad \forall t \in\left[t_{1}, \tau\right) .
$$

Similarly, the third equation of (1.2) yields

$$
\begin{aligned}
R(x, t) & =\Gamma_{3}(t) R\left(x, t_{1}\right)+\int_{t_{1}}^{t} \Gamma_{3}(t-s) \gamma_{2}(x) I(x, t) \mathrm{d} s \\
& \leq M_{3} e^{\alpha_{3}\left(t-t_{1}\right)}\left\|R\left(\cdot, t_{1}\right)\right\|+\int_{t_{1}}^{t} \int_{\Omega} T_{3}(x, y, t-s) \gamma_{2}(x) I(x, t) \mathrm{d} y \mathrm{~d} s \\
& \leq M_{3} e^{\alpha_{3}\left(t-t_{1}\right)}\left\|R\left(\cdot, t_{1}\right)\right\|+w_{3} \gamma_{2}^{+} N_{1} \frac{1-e^{-\left(t-t_{1}\right) \pi_{3}^{-}}}{\pi_{3}^{-}} \\
& \leq M_{3} e^{-\alpha_{3} t_{1}}\left\|R\left(\cdot, t_{1}\right)\right\|+\frac{w_{3} \gamma_{2}^{+} N_{1}}{\mu^{-}+\delta^{-}}, \quad \forall t \in[0, \tau),
\end{aligned}
$$

and thus,

$$
\|R(\cdot, t)\| \leq M_{3} e^{-\alpha_{3} t_{1}}\left\|R\left(\cdot, t_{1}\right)\right\|+\frac{w_{3} \gamma_{2}^{+} N_{1}}{\mu^{-}+\delta^{-}}, \quad \forall t \in\left[t_{1}, \tau\right)
$$


Hence, we conclude that $S, I$ and $R$ are bounded on $\Omega \times[0, \tau)$, which contradicts $(2.3)$. This proves that $\tau=+\infty$.

In a similar way, we can obtain the following corollary on the boundedness of solutions on $[0, \infty)$.

Corollary 2.2. For each solution $U(\cdot, t ; \phi)=(S(\cdot, t ; \phi), I(\cdot, t ; \phi), R(\cdot, t ; \phi))$ of (1.2) with initial value function $\phi \in \mathbb{X}^{+}$, there exist positive constants $M_{S}, M_{I}, M_{R}$ independent of initial data such that

$$
\limsup _{t \rightarrow \infty}\|S(\cdot, t)\| \leq M_{S}, \quad \limsup _{t \rightarrow \infty}\|I(\cdot, t)\| \leq M_{I}, \quad \limsup _{t \rightarrow \infty}\|R(\cdot, t)\| \leq M_{R} .
$$

Furthermore, the solution semiflow $\Phi_{t}: \mathbb{X}^{+} \rightarrow \mathbb{X}^{+}$is point dissipative and has a global compact attractor.

Proof. Using the same notation as in the proof of Theorem 2.1 and replacing $\tau$ by $+\infty$, we can show that

$$
M_{S}=\frac{w_{1}\left(\Lambda^{+}+\gamma_{1}^{+} N_{1}+\delta^{+} N_{1}\right)}{\mu^{-}}, \quad M_{I}=\frac{w_{2} \beta^{+} N_{1} M_{S}}{\mu^{-}+\alpha^{-}+\gamma_{1}^{-}+\gamma_{2}^{-}}, \quad M_{R}=\frac{w_{3} \gamma_{2}^{+} N_{1}}{\mu^{-}+\delta^{-}}
$$

satisfy (2.5). From this, it follows that the system is point dissipative. In addition, we know by [26, Theorem 2.2.6] that the solution semiflow $\Phi_{t}$ is compact for any $t>0$. Then, we have by [27, Theorem 3.4.8] that $\Phi_{t}$ has a global compact attractor. Consequently, the proof is complete.

\section{Basic reproduction number and stability of the disease-free steady state}

In this section, we will study the disease-free dynamics of model (1.2) and determine the basic reproduction number $\mathcal{R}_{0}$, which is defined as the average number of secondary infections generated by a single infected individual introduced in a completely susceptible population.

Setting $I(x, t)=R(x, t) \equiv 0$ in (1.2), we obtain the following equation for the density of susceptible population in absence of the disease:

$$
\begin{array}{ll}
\partial_{t} S=\nabla \cdot\left(d_{S}(x) \nabla S\right)+\Lambda(x)-\mu(x) S, & x \in \Omega, t>0, \\
{\left[d_{S}(x) \nabla S(x, t)\right] \cdot \mathbf{n}=0,} & x \in \partial \Omega, t>0,
\end{array}
$$

System (3.1) admits a unique positive steady state $S_{0}(x)$, which is globally asymptotically stable (see [20, Lemma 1]). We will call $E_{0}:=\left(S_{0}(\cdot), 0,0\right) \in \mathbb{X}^{+}$the disease-free steady state of model $(1.2)$.

Linearizing the equation for the infected population in (1.2) around $E_{0}$, we obtain

$$
\begin{array}{ll}
\partial_{t} I=\nabla \cdot\left(d_{I}(x) \nabla I\right)+\left[\frac{\partial F}{\partial I}\left(x, S_{0}, 0\right)-\left(\mu+\alpha+\gamma_{1}+\gamma_{2}\right)(x)\right] I, & x \in \Omega, t>0, \\
{\left[d_{I}(x) \nabla I(x, t)\right] \cdot \mathbf{n}=0,} & x \in \partial \Omega, t>0 .
\end{array}
$$

Substituting $I(x, t)=e^{\lambda t} \varphi(x)$ in the above equation yields the following eigenvalue problem:

$$
\begin{array}{ll}
\lambda \varphi(x)=\nabla \cdot\left(d_{I}(x) \nabla \varphi(x)\right)+\left[\frac{\partial F}{\partial I}\left(x, S_{0}, 0\right)-\left(\mu+\alpha+\gamma_{1}+\gamma_{2}\right)(x)\right] \varphi(x), & x \in \Omega \\
{\left[d_{I}(x) \nabla \varphi(x)\right] \cdot \mathbf{n}=0,} & x \in \partial \Omega
\end{array}
$$

It follows from the standard Krein-Rutman theorem that (3.3) has a principal eigenvalue $\lambda^{*}\left(S_{0}\right)=$ $\mathbf{s}\left(\nabla \cdot\left(d_{I} \nabla\right)+\frac{\partial F}{\partial I}\left(\cdot, S_{0}, 0\right)-\left(\mu+\alpha+\gamma_{1}+\gamma_{2}\right)\right)$ corresponding to a positive eigenfunction $\varphi^{*}(x)$, where $\mathbf{s}(A)$ denotes the spectral bound of a closed linear operator $A$.

It is well-known that $\lambda^{*}\left(S_{0}\right)$ is given by the variational characterization

$$
\begin{gathered}
\lambda^{*}\left(S_{0}\right)=-\inf \left\{\int_{\Omega}\left[d_{I}(x)|\nabla \varphi(x)|^{2}+\left[\left(\mu+\alpha+\gamma_{1}+\gamma_{2}\right)(x)-\frac{\partial F}{\partial I}\left(x, S_{0}, 0\right)\right] \varphi^{2}(x)\right] \mathrm{d} x:\right. \\
\left.\varphi \in H^{1}(\Omega) \text { and } \int_{\Omega} \varphi^{2}(x) \mathrm{d} x=1\right\} .
\end{gathered}
$$


Let $\mathcal{T}_{2}(t)$ be the semigroup on $C(\bar{\Omega}, \mathbb{R})$ associated with $\nabla \cdot\left(d_{I} \nabla I\right)-\left(\mu+\alpha+\gamma_{1}+\gamma_{2}\right)$. In order to define the basic reproduction number for our model, we assume that the population is near the diseasefree steady state $E_{0}$, and introduce infected individuals at time $t=0$, where the spatial distribution of infected population is described by $\phi_{2}(x)$. Then, $\mathcal{T}_{2}(t) \phi_{2}(x)$ is the distribution of infected population as time evolves. It follows that the distribution of new infections at time $t$ is $\frac{\partial F}{\partial I}\left(x, S_{0}, 0\right) \mathcal{T}_{2}(t) \phi_{2}(x)$. Thus, the distribution of total new infections becomes

$$
\mathcal{L}\left(\phi_{2}\right)(x):=\int_{0}^{\infty} \frac{\partial F}{\partial I}\left(x, S_{0}, 0\right) \mathcal{T}_{2}(t) \phi_{2}(x) \mathrm{d} t=\frac{\partial F}{\partial I}\left(x, S_{0}, 0\right) \int_{0}^{\infty} \mathcal{T}_{2}(t) \phi_{2}(x) \mathrm{d} t,
$$

where $\mathcal{L}$ is the next infection operator, which maps the initial distribution $\phi_{2}$ of infected individuals to the distribution of total infected individuals produced during the infection period.

Following [28-31], we define the basic reproduction number for model (1.2) as the spectral radius of $\mathcal{L}$, that is,

$$
\mathcal{R}_{0}:=\rho(\mathcal{L})=\sup _{0 \neq \varphi \in H^{1}(\Omega)}\left\{\frac{\int_{\Omega} \frac{\partial F}{\partial I}\left(x, S_{0}, 0\right) \varphi^{2}(x) \mathrm{d} x}{\int_{\Omega}\left[d_{I}(x)|\nabla \varphi(x)|^{2}+\left(\mu+\alpha+\gamma_{1}+\gamma_{2}\right)(x) \varphi^{2}(x)\right] \mathrm{d} x}\right\} .
$$

Define $u=(I, S, R)$,

$$
\mathcal{F}(x, u)=\left(\begin{array}{c}
F(x, S, I) \\
0 \\
0
\end{array}\right), \quad \mathcal{V}(x, u)=\left(\begin{array}{c}
\left(\mu+\alpha+\gamma_{1}+\gamma_{2}\right)(x) I \\
\mu(x) S+F(x, S, I)-\Lambda(x)-\gamma_{1}(x) I-\delta(x) R \\
\mu(x) R+\delta(x) R-\gamma_{2}(x) I
\end{array}\right)
$$

Thus, model (1.2) can be rewritten as

$$
\begin{array}{ll}
\partial_{t} u_{i}=\nabla \cdot\left(d_{i}(x) \nabla u_{i}\right)+\mathcal{F}_{i}(x, u)-\mathcal{V}_{i}(x, u), & 1 \leq i \leq 3, x \in \Omega, t>0, \\
{\left[d_{i}(x) \nabla u_{i}(x, t)\right] \cdot \mathbf{n}=0,} & 1 \leq i \leq 3, x \in \partial \Omega, t>0,
\end{array}
$$

where $d_{1}=d_{I}, d_{2}=d_{S}$ and $d_{3}=d_{R}$. The disease-free steady state is given by $u_{0}(x)=\left(0, S_{0}(x), 0\right)$, with the variables ordered as $(I, S, R)$.

We can immediately verify that assumptions (A1)-(A4) in [31] hold. Moreover, if we define

$$
M^{0}(x)=\left(\begin{array}{cc}
-\mu(x) & \delta(x) \\
0 & -\mu(x)-\delta(x)
\end{array}\right), \quad F_{0}(x)=\frac{\partial F}{\partial I}\left(x, S_{0}, 0\right) \quad \text { and } \quad V(x)=\left(\mu+\alpha+\gamma_{1}+\gamma_{2}\right)(x),
$$

then $M^{0}(x)$ and $-V(x)$ are cooperative matrices for all $x \in \bar{\Omega}$, i.e. their off-diagonal elements are nonnegative. Hence, assumptions (A5) and (A6) in [31] also hold, so we can conclude the following result by [31, Theorem 3.1].

Lemma 3.1. $\mathcal{R}_{0}-1$ has the same sign as $\lambda^{*}$. Furthermore, if $\mathcal{R}_{0}<1$, then $E_{0}$ is locally asymptotically stable for system (1.2).

Before proving the main result of this section, we give the following lemma.

Lemma 3.2. Suppose that $U(\cdot, t ; \phi)=(S(\cdot, t ; \phi), I(\cdot, t ; \phi), R(\cdot, t ; \phi))$ is the solution of system $(1.2)$ with $U(\cdot, 0 ; \phi)=\phi \in \mathbb{X}^{+}$. Then:

(i) For any $\phi \in \mathbb{X}^{+}$, we always have $S(x, t ; \phi)>0$ for all $t>0, x \in \bar{\Omega}$, and

$$
\liminf _{t \rightarrow \infty} S(x, t ; \phi) \geq \frac{\Lambda^{-}}{\mu^{+}+\beta^{+} M_{I}}, \quad \text { uniformly for } x \in \bar{\Omega} .
$$

(ii) If there exists $t_{0} \geq 0$ such that $I\left(\cdot, t_{0} ; \phi\right) \not \equiv 0$ (respectively, $\left.R\left(\cdot, t_{0} ; \phi\right) \not \equiv 0\right)$, then, for $t>t_{0}$, we have $I(\cdot, t ; \phi)>0$ (respectively, $R(\cdot, t ; \phi)>0)$. 
Proof. By assumption (A4) and Corollary 2.2, we have $F(x, S, I) \leq \beta^{+} S I \leq \beta^{+} M_{I} S$ for all $x \in \Omega$, $S, I \geq 0$. Then, from the first equation of (1.2), we get

$$
\begin{array}{ll}
\partial_{t} S \geq \nabla \cdot\left(d_{S}(x) \nabla S\right)+\Lambda^{-}-\left(\mu^{+}+\beta^{+} M_{I}\right) S, & x \in \Omega, t>0, \\
{\left[d_{S}(x) \nabla S(x, t)\right] \cdot \mathbf{n}=0,} & x \in \partial \Omega, t>0 .
\end{array}
$$

By the comparison principle, this shows that $\liminf _{t \rightarrow \infty} S(x, t ; \phi) \geq \Lambda^{-} /\left(\mu^{+}+\beta^{+} M_{I}\right)$ uniformly for $x \in \bar{\Omega}$.

From system (1.2), we can also get

$$
\begin{array}{ll}
\partial_{t} I \geq \nabla \cdot\left(d_{I}(x) \nabla I\right)-\left(\mu+\alpha+\gamma_{1}+\gamma_{2}\right)(x) I, & x \in \Omega, t>0, \\
{\left[d_{I}(x) \nabla I(x, t)\right] \cdot \mathbf{n}=0,} & x \in \partial \Omega, t>0 .
\end{array}
$$

and

$$
\begin{array}{ll}
\partial_{t} R \geq \nabla \cdot\left(d_{R}(x) \nabla R\right)-(\mu+\delta)(x) R, & x \in \Omega, t>0, \\
{\left[d_{R}(x) \nabla R(x, t)\right] \cdot \mathbf{n}=0,} & x \in \partial \Omega, t>0 .
\end{array}
$$

Using the strong maximum principle (see [32, Proposition 13.1]), part (ii) of the lemma is concluded.

Next, we give the main result concerning the stability of $E_{0}$ in terms of the basic reproduction number.

Theorem 3.3. Assume that $U(\cdot, t ; \phi)$ is the solution of system $(1.2)$ with $U(\cdot, 0 ; \phi)=\phi \in \mathbb{X}^{+}$. Then, the following statements are valid.

(i) If $\mathcal{R}_{0}<1$, then the disease-free steady state $E_{0}=\left(S_{0}, 0,0\right)$ is globally asymptotically stable.

(ii) If $\mathcal{R}_{0}>1$, then there exists a constant $\varepsilon_{0}>0$ such that any positive solution of (1.2) satisfies

$$
\limsup _{t \rightarrow \infty}\left\|(S(\cdot, t), I(\cdot, t), R(\cdot, t))-\left(S_{0}, 0,0\right)\right\|>\varepsilon_{0} .
$$

Proof. (i) By Lemma 3.1, we have $\lambda^{*}\left(S_{0}\right)<0$ when $\mathcal{R}_{0}<1$. Thus, there exists a sufficiently small $\varepsilon$ such that $\lambda^{*}\left(S_{0}+\varepsilon\right)<0$. According to Corollary 2.2, there exists a $\tau_{1}>0$ such that

$$
S(x, t) \leq S_{0}(x)+\varepsilon, \quad \text { for all } t \geq \tau_{1}, x \in \Omega .
$$

It follows from (A1)-(A3) that

$$
\begin{aligned}
F(x, S, I) & =I F_{1}(x, S, I) \leq I F_{1}\left(x, S_{0}(x)+\varepsilon, I\right) \leq I \lim _{h \rightarrow 0} F_{1}\left(x, S_{0}(x)+\varepsilon, h\right) \\
& =I \lim _{h \rightarrow 0} \frac{F\left(x, S_{0}(x)+\varepsilon, h\right)-F\left(x, S_{0}(x)+\varepsilon, 0\right)}{h} \\
& =I \frac{\partial F}{\partial I}\left(x, S_{0}(x)+\varepsilon, 0\right)
\end{aligned}
$$

for $t \geq \tau_{1}$. Hence, by the second equation of (1.2), we have

$$
\begin{array}{ll}
\partial_{t} I \leq \nabla \cdot\left(d_{I}(x) \nabla I\right)+\left[\frac{\partial F}{\partial I}\left(x, S_{0}(x)+\varepsilon, 0\right)-\left(\mu+\alpha+\gamma_{1}+\gamma_{2}\right)(x)\right] I, & x \in \Omega, t \geq \tau_{1}, \\
{\left[d_{I}(x) \nabla I(x, t)\right] \cdot \mathbf{n}=0,} & x \in \partial \Omega, t \geq \tau_{1} .
\end{array}
$$

Let $\psi(x)$ be the eigenfunction corresponding to the principal eigenvalue $\lambda^{*}\left(S_{0}+\varepsilon\right)<0$. Let $\xi_{1}>0$ be such that $I\left(x, \tau_{1}\right) \leq \xi_{1} \psi(x)$. By the comparison principle, we get

$$
I(x, t) \leq \xi_{1} \psi(x) e^{\lambda^{*}\left(S_{0}(x)+\varepsilon\right)\left(t-\tau_{1}\right)}, \quad \text { for all } t \geq \tau_{1}, x \in \Omega .
$$

This yields that $\lim _{t \rightarrow \infty} I(x, t)=0$ uniformly for $x \in \bar{\Omega}$. Then, the third equation of (1.2) is asymptotic to

$$
\partial_{t} R=\nabla \cdot\left(d_{R}(x) \nabla R\right)-(\mu+\delta)(x) R,
$$

so $\lim _{t \rightarrow \infty} R(x, t)=0$ uniformly for $x \in \bar{\Omega}$. Moreover, the first equation of (1.2) is asymptotic to

$$
\partial_{t} S=\nabla \cdot\left(d_{S}(x) \nabla S\right)+\Lambda(x)-\mu(x) S,
$$

which implies that $\lim _{t \rightarrow \infty} S(x, t)=S_{0}(x)$ uniformly for $x \in \bar{\Omega}$. This completes the proof. 
(ii) Assume by contradiction that there exists a positive solution of (1.2) such that

$$
\limsup _{t \rightarrow \infty}\left\|(S(\cdot, t), I(\cdot, t), R(\cdot, t))-\left(S_{0}, 0,0\right)\right\|<\varepsilon_{0} .
$$

Then, there exists $t_{1}>0$ such that $S_{0}-\varepsilon_{0}<S(x, t)<S_{0}+\varepsilon_{0}$ and $0<I(x, t)<\varepsilon_{0}$ for $t \geq t_{1}$. It follows from (A1)-(A3) that

$$
\frac{F(x, S, I)}{I}=F_{1}(x, S, I) \geq F_{1}\left(x, S_{0}-\varepsilon_{0}, \varepsilon_{0}\right) \geq \frac{\partial F}{\partial I}\left(x, S_{0}-\varepsilon_{0}, \varepsilon_{0}\right) .
$$

Therefore, $I(x, t ; \phi)$ satisfies

$$
\begin{array}{ll}
\partial_{t} I \geq \nabla \cdot\left(d_{I}(x) \nabla I\right)+\left[\frac{\partial F}{\partial I}\left(x, S_{0}-\varepsilon_{0}, \varepsilon_{0}\right)-\left(\mu+\alpha+\gamma_{1}+\gamma_{2}\right)(x)\right] I, & x \in \Omega, t \geq t_{1}, \\
{\left[d_{I}(x) \nabla I(x, t)\right] \cdot \mathbf{n}=0,} & x \in \partial \Omega, t \geq t_{1} .
\end{array}
$$

For any $\varepsilon \in\left(0, \min _{x \in \bar{\Omega}} S_{0}(x)\right)$, we consider the eigenvalue problem

$$
\begin{array}{ll}
\lambda \varphi(x)=\nabla \cdot\left(d_{I}(x) \nabla \varphi(x)\right)+\left[\frac{\partial F}{\partial I}\left(x, S_{0}-\varepsilon, \varepsilon\right)-\left(\mu+\alpha+\gamma_{1}+\gamma_{2}\right)(x)\right] \varphi(x), & x \in \Omega, \\
{\left[d_{I}(x) \nabla \varphi(x)\right] \cdot \mathbf{n}=0,} & x \in \partial \Omega .
\end{array}
$$

Define $\mathcal{R}_{\varepsilon}$ as the spectral radius of the operator

$$
\mathcal{L}_{\varepsilon}: \phi \rightarrow \frac{\partial F}{\partial I}\left(\cdot, S_{0}-\varepsilon, \varepsilon\right) \int_{0}^{\infty} \mathcal{T}_{2}(t) \phi \mathrm{d} t .
$$

Since $\lim _{\varepsilon \rightarrow 0} \mathcal{R}_{\varepsilon}=\mathcal{R}_{0}>1$, we restrict $\varepsilon$ to be small enough such that $\mathcal{R}_{\varepsilon}>1$. Hence $\lambda_{\varepsilon}^{*}:=$ $\mathbf{s}\left(\nabla \cdot\left(d_{I} \nabla\right)+\frac{\partial F}{\partial I}\left(\cdot, S_{0}-\varepsilon, \varepsilon\right)-\left(\mu+\alpha+\gamma_{1}+\gamma_{2}\right)\right)>0$. As a consequence, we can fix a small $\varepsilon_{0} \in\left(\varepsilon, \min _{x \in \bar{\Omega}} S_{0}(x)\right)$ such that $\lambda_{\varepsilon_{0}}^{*}>0$.

By assumption, $I(x, t)>0$ for all $x \in \bar{\Omega}$ and $t>0$. Then, by Lemma 3.2(ii), we can choose a sufficiently small number $\eta>0$ such that $I(\cdot, t) \geq \eta \phi_{\varepsilon_{0}}^{*}(\cdot)$, where $\phi_{\varepsilon_{0}}^{*}(\cdot)$ is a strongly positive eigenfunction corresponding to $\lambda_{\varepsilon_{0}}^{*}$. Notice that $u_{1}(x, t):=\eta \exp \left(\lambda_{\varepsilon_{0}}^{*}\left(t-t_{1}\right)\right) \phi_{\varepsilon_{0}}^{*}(x)$ is a solution to the linear system

$$
\begin{array}{ll}
\partial_{t} I=\nabla \cdot\left(d_{I}(x) \nabla I\right)+\left[\frac{\partial F}{\partial I}\left(x, S_{0}-\varepsilon_{0}, \varepsilon_{0}\right)-\left(\mu+\alpha+\gamma_{1}+\gamma_{2}\right)(x)\right] I, & x \in \Omega, t \geq t_{1}, \\
{\left[d_{I}(x) \nabla I(x, t)\right] \cdot \mathbf{n}=0,} & x \in \partial \Omega, t \geq t_{1} .
\end{array}
$$

It then follows from (3.11) and the comparison principle that

$$
I(x, t) \geq \eta \exp \left(\lambda_{\varepsilon_{0}}^{*}\left(t-t_{1}\right)\right) \phi_{\varepsilon_{0}}^{*}(x), \quad \text { for all } x \in \Omega, t \geq t_{1} .
$$

Since $\lambda_{\varepsilon_{0}}^{*}>0$, this implies that $I(x, t)$ is unbounded, which is a contradiction.

\section{Uniform persistence}

We will now study the existence and persistence of the endemic equilibrium for model (1.2).

Theorem 4.1. Suppose that $\mathcal{R}_{0}>1$. Then, (1.2) is uniformly persistent in the sense that there exists a constant $\varepsilon>0$ such that for any $\phi \in \mathbb{X}^{+}$with $\phi_{2}(\cdot) \not \equiv 0$, we have

$$
\liminf _{t \rightarrow \infty} S(x, t ; \phi) \geq \varepsilon, \quad \liminf _{t \rightarrow \infty} I(x, t ; \phi) \geq \varepsilon, \quad \liminf _{t \rightarrow \infty} R(x, t ; \phi) \geq \varepsilon, \quad \text { uniformly for } x \in \bar{\Omega} .
$$

Moreover, (1.2) admits at least one endemic equilibrium $\left(S^{*}, I^{*}, R^{*}\right)$. 
Proof. Let

$$
\mathbb{W}_{0}=\left\{\left(\phi_{1}, \phi_{2}, \phi_{3}\right) \in \mathbb{X}^{+}: \phi_{2} \not \equiv 0 \text { and } \phi_{3} \not \equiv 0\right\}
$$

and

$$
\partial \mathbb{W}_{0}=\mathbb{X}^{+} \backslash \mathbb{W}_{0}=\left\{\left(\phi_{1}, \phi_{2}, \phi_{3}\right) \in \mathbb{X}^{+}: \phi_{2} \equiv 0 \text { or } \phi_{3} \equiv 0\right\} .
$$

By Lemma 3.2(ii), it follows that for any $\phi \in \mathbb{W}_{0}$, we have $I(x, t ; \phi)>0$ and $R(x, t ; \phi)>0$ for all $x \in \bar{\Omega}$, $t>0$. Hence, $\Phi_{t} \mathbb{W}_{0} \subseteq \mathbb{W}_{0}$ for all $t \geq 0$.

Define

$$
\mathbb{M}_{\partial}=\left\{\phi \in \partial \mathbb{W}_{0}: \Phi_{t}(\phi) \in \partial \mathbb{W}_{0} \text { for all } t \geq 0\right\}
$$

and let $\omega(\phi)$ be the omega limit set of the orbit $O^{+}(\phi):=\left\{\Phi_{t}(\phi): t \geq 0\right\}$. We will now show that $\omega(\phi)=\left\{\left(S_{0}(\cdot), 0,0\right)\right\}$ for all $\phi \in M_{\partial}$.

For any given $\phi \in \mathbb{M}_{\partial}$, we have $\Phi_{t}(\phi) \in \partial \mathbb{W}_{0}$. It then follows that for each $t \geq 0$, either $I(\cdot, t ; \phi) \equiv 0$ or $R(\cdot, t ; \phi) \equiv 0$. In the case where $I(\cdot, t ; \phi) \equiv 0$, we can see that $R(x, t ; \phi)$ satisfies

$$
\begin{array}{ll}
\partial_{t} R=\nabla \cdot\left(d_{R}(x) \nabla R\right)-(\mu+\delta)(x) R, & x \in \Omega, t>0, \\
{\left[d_{R}(x) \nabla R(x, t)\right] \cdot \mathbf{n}=0,} & x \in \partial \Omega, t>0 .
\end{array}
$$

which implies that $\lim _{t \rightarrow \infty} R(\cdot, t ; \phi)=0$ uniformly for $x \in \bar{\Omega}$. Thus, for any sufficiently small $\varepsilon>0$, there is a $t_{2}>0$ such that $R(x, t ; \phi)<\varepsilon$ for $t \geq t_{2}$. Then, from the first equation of (1.2), we can get

$$
\begin{array}{ll}
\partial_{t} S=\nabla \cdot\left(d_{S}(x) \nabla S\right)+\Lambda(x)-\mu(x) S+\varepsilon \delta(x), & x \in \Omega, t>t_{2}, \\
{\left[d_{S}(x) \nabla S(x, t)\right] \cdot \mathbf{n}=0,} & x \in \partial \Omega, t>t_{2} .
\end{array}
$$

Since $\varepsilon$ is arbitrary, this shows that $\lim _{t \rightarrow \infty} S(x, t ; \phi)=S_{0}(x)$. On the other hand, when $I\left(\cdot, t_{3} ; \phi\right) \not \equiv 0$ for some $t_{3} \geq 0$, we obtain by Lemma 3.2 (ii) that $I(x, t ; \phi)>0$ for all $x \in \Omega, t>t_{3}$. Hence, $R(\cdot, t ; \phi) \equiv 0$ for all $t>t_{3}$, but the last equation of (1.2) implies that $I(\cdot, t ; \phi) \equiv 0$ for $t>t_{3}$, which is a contradiction. Thus, we have proved that $\omega(\phi)=\left\{\left(S_{0}(\cdot), 0,0\right)\right\}$ for all $\phi \in M_{\partial}$. Moreover, since $\mathcal{R}_{0}>1$, it follows from Theorem 3.3(ii) that $E_{0}=\left(S_{0}, 0,0\right)$ is a uniform weak repeller for $\mathbb{W}_{0}$.

Define a continuous function $p: \mathbb{X}^{+} \rightarrow[0, \infty)$ by

$$
p(\phi):=\min \left\{\min _{x \in \bar{\Omega}} \phi_{2}(x), \min _{x \in \bar{\Omega}} \phi_{3}(x)\right\} \quad \text { for } \phi=\left(\phi_{1}, \phi_{2}, \phi_{3}\right) \in \mathbb{X}^{+} .
$$

It follows from Lemma 3.2(ii) that $p^{-1}(0, \infty) \subseteq \mathbb{W}_{0}$, and $p$ has the property that if $p(\phi)>0$ or $\phi \in \mathbb{W}_{0}$ with $p(\phi)=0$, then $p\left(\Phi_{t}(\phi)\right)>0$ for all $t>0$. Thus, $p$ is a generalized distance function for the semiflow $\Phi_{t}: \mathbb{X}^{+} \rightarrow \mathbb{X}^{+}$(see [33]).

Note that any forward orbit of $\Phi_{t}$ in $M_{\partial}$ converges to $E_{0}$. Furthermore, the claim above implies that $E_{0}$ is isolated in $\mathbb{X}^{+}$and $W^{S}\left(E_{0}\right) \cap \mathbb{W}_{0}=\emptyset$, where $W^{S}\left(E_{0}\right)$ is the stable set of $E_{0}$. Moreover, it is clear that there are no cycles in $M_{\partial}$ from $E_{0}$ to $E_{0}$. By [33, Theorem 3], it follows that there exists a constant $\eta_{1}>0$ such that $\min \{p(\psi): \psi \in \omega(\phi)\}>\eta_{1}$ for any $\phi \in \mathbb{W}_{0}$. Hence,

$$
\liminf _{t \rightarrow \infty} I(\cdot, t ; \phi), \liminf _{t \rightarrow \infty} R(\cdot, t ; \phi) \geq \eta_{1} \quad \text { for all } \phi \in \mathbb{W}_{0} .
$$

Further, it follows from Lemma 3.2(i) that

$$
\liminf _{t \rightarrow \infty} S(\cdot, t ; \phi) \geq \frac{\Lambda^{-}}{\mu^{+}+\beta^{+} M_{I}}=: \eta_{2} \quad \text { for all } \phi \in \mathbb{W}_{0} .
$$

Thus, (4.1) holds by taking $\varepsilon=\min \left\{\eta_{1}, \eta_{2}\right\}$, which proves the uniform persistence result. By Theorem 3.7 and Remark 3.10 in [34], it follows that $\Phi_{t}: \mathbb{W}_{0} \rightarrow \mathbb{W}_{0}$ has a global attractor. It then follows from [34, Theorem 4.7] that $\Phi_{t}$ has a steady state $\left(S^{*}, I^{*}, R^{*}\right) \in \mathbb{W}_{0}$, which is positive by Lemma 3.2(ii). Therefore, $\left(S^{*}, I^{*}, R^{*}\right)$ is an endemic equilibrium of model (1.2). 


\section{Bifurcation analysis}

In this section, we will use $\gamma_{1}$ (the transfer rate from the infected class to the susceptible class) as the main bifurcation parameter to perform the bifurcation analysis of model (1.2). To apply the local and global bifurcation theory by Crandall and Rabinowitz [35], we need to assume that the diffusion coefficient $d_{I}$ and the parameter $\gamma_{1}$ are constant, where $d_{I}>0$ and $\gamma_{1} \geq 0$. However, we still allow all other parameters and the diffusion rates $d_{S}(x)$ and $d_{R}(x)$ to be spatially variable.

A steady state of model (1.2) is a solution of the elliptic problem

$$
\begin{array}{lll}
\nabla \cdot\left(d_{S}(x) \nabla \tilde{S}(x)\right)+\Lambda(x)-\mu(x) \tilde{S}(x)-F(x, \tilde{S}(x), \tilde{I}(x))+\gamma_{1}(x) \tilde{I}(x) & & \\
\quad+\delta(x) \tilde{R}(x)=0, & & x \in \Omega, \\
\nabla \cdot\left(d_{I}(x) \nabla \tilde{I}(x)\right)+F(x, \tilde{S}(x), \tilde{I}(x))-\left(\mu+\alpha+\gamma_{1}+\gamma_{2}\right)(x) \tilde{I}(x)=0, & x \in \Omega, \\
\nabla \cdot\left(d_{R}(x) \nabla \tilde{R}(x)\right)+\gamma_{2}(x) \tilde{I}(x)-\mu(x) \tilde{R}(x)-\delta(x) \tilde{R}(x)=0, & & x \in \Omega, \\
{\left[d_{S}(x) \nabla \tilde{S}(x)\right] \cdot \mathbf{n}=\left[d_{I}(x) \nabla \tilde{I}(x)\right] \cdot \mathbf{n}=\left[d_{R}(x) \nabla \tilde{R}(x)\right] \cdot \mathbf{n}=0,} & & x \in \partial \Omega .
\end{array}
$$

When $d_{I}(x)=d_{I}$ and $\gamma_{1}(x)=\gamma_{1}$ are constant, system (5.1) becomes

$$
\begin{aligned}
& \nabla \cdot\left(d_{S}(x) \nabla \tilde{S}(x)\right)+\Lambda(x)-\mu(x) \tilde{S}(x)-F(x, \tilde{S}(x), \tilde{I}(x))+\gamma_{1} \tilde{I}(x) \\
& +\delta(x) \tilde{R}(x)=0, \quad x \in \Omega, \\
& d_{I} \Delta \tilde{I}(x)+F(x, \tilde{S}(x), \tilde{I}(x))-\left(\mu+\alpha+\gamma_{2}\right)(x) \tilde{I}(x)-\gamma_{1} \tilde{I}(x)=0, \quad x \in \Omega, \\
& \nabla \cdot\left(d_{R}(x) \nabla \tilde{R}(x)\right)+\gamma_{2}(x) \tilde{I}(x)-\mu(x) \tilde{R}(x)-\delta(x) \tilde{R}(x)=0, \quad x \in \Omega \text {, } \\
& {\left[d_{S}(x) \nabla \tilde{S}(x)\right] \cdot \mathbf{n}=\left[d_{I} \nabla \tilde{I}(x)\right] \cdot \mathbf{n}=\left[d_{R}(x) \nabla \tilde{R}(x)\right] \cdot \mathbf{n}=0, \quad x \in \partial \Omega .}
\end{aligned}
$$

It is easy to see that $\left(S_{0}(\cdot), 0,0\right)$ is a semi-trivial steady state solution of (5.2). Denote by $\gamma_{1}^{*}$ the principal eigenvalue of the eigenvalue problem

$$
\begin{array}{ll}
d_{I} \Delta \psi(x)+\left[\frac{\partial F}{\partial I}\left(x, S_{0}(x), 0\right)-\left(\mu+\alpha+\gamma_{2}\right)(x)\right] \psi(x)=\gamma_{1} \psi(x), & x \in \Omega, \\
{\left[d_{I} \nabla \psi(x)\right] \cdot \mathbf{n}=0,} & x \in \partial \Omega,
\end{array}
$$

associated with a positive eigenfunction $\psi_{0}(x)$, which is uniquely determined by the normalization $\max _{x \in \bar{\Omega}} \psi_{0}(x)=1$. By expression (3.5), we know that $\mathcal{R}_{0}$ is decreasing with respect to $\gamma_{1}$. Furthermore, (5.3) with $\gamma_{1}=\gamma_{1}^{*}$ is equivalent to system (3.3) with $\gamma_{1}(x)=\gamma_{1}^{*}, d_{I}(x)=d_{I}$ and $\lambda=0$, and by Lemma 3.1, we have that $\lambda^{*}=0$ if and only if $\mathcal{R}_{0}=1$. This shows that the condition $\gamma_{1}=\gamma_{1}^{*}$ is equivalent to $\mathcal{R}_{0}=1$.

Next, we define a function

$$
H(x)=\frac{\partial F}{\partial I}\left(x, S_{0}(x), 0\right)-\left(\mu+\alpha+\gamma_{2}\right)(x) .
$$

Then, it follows that $\gamma_{1}^{*}=H$ when $H$ is a constant.

We will now study the case where $H$ is not constant and it could change sign in $\Omega$. Consider the eigenvalue problem with indefinite weight

$$
\begin{array}{ll}
\Delta \varphi(x)+\Lambda H(x) \varphi(x)=0, & x \in \Omega, \\
{[\nabla \varphi(x)] \cdot \mathbf{n}=0,} & x \in \partial \Omega .
\end{array}
$$

It follows from [36, Theorem 4.2] that the problem (5.5) has a nonzero principal eigenvalue $\Lambda_{0}=\Lambda_{0}(H)$ if and only if $H(x)$ changes sign in $\Omega$ and $\int_{\Omega} H(x) \mathrm{d} x \neq 0$.

It then follows from [36, Proposition 4.4] that the sign of the principal eigenvalue $\gamma_{1}^{*}$ of the problem (5.3) is described by the following result.

Lemma 5.1. The following statements hold.

(i) If $\int_{\Omega} H(x) \mathrm{d} x \geq 0$, then $\gamma_{1}^{*}>0$ for all $d_{I}>0$. 
(ii) If $\int_{\Omega} H(x) \mathrm{d} x<0$, then

$$
\left\{\begin{array}{l}
\gamma_{1}^{*}>0 \text { for all } d_{I}<\frac{1}{\Lambda_{0}(H)} \\
\gamma_{1}^{*}<0 \text { for all } d_{I}>\frac{1}{\Lambda_{0}(H)}
\end{array} .\right.
$$

We next regard $\gamma_{1}$ as a bifurcation parameter and investigate a local branch of positive solutions of (5.2) that bifurcates from the branch of semi-trivial solutions $\left\{\left(S_{0}(\cdot), 0,0, \gamma_{1}\right): \gamma_{1} \geq 0\right\}$. We note that $S_{0}(\cdot)$ is independent of the parameter $\gamma_{1}$.

Theorem 5.2. Let

$$
\mathcal{S}=\left\{\left(S, I, R, \gamma_{1}\right) \in X \times \mathbb{R}_{+}:\left(S, I, R, \gamma_{1}\right)=\left(S_{0}(\cdot), 0,0, \gamma_{1}\right)\right\},
$$

be the set of semi-trivial solutions of (5.2), where

$$
\begin{aligned}
X=\{ & (u, v, w) \in W^{2, p}(\Omega) \times W^{2, p}(\Omega) \times W^{2, p}(\Omega): \\
& {\left.\left[d_{S}(x) \nabla u(x)\right] \cdot \mathbf{n}=\left[d_{I} \nabla v(x)\right] \cdot \mathbf{n}=\left[d_{R}(x) \nabla w(x)\right] \cdot \mathbf{n}=0, \forall x \in \partial \Omega\right\} }
\end{aligned}
$$

for $p>n$. Then, a branch of positive solutions of (5.2) bifurcates from $\mathcal{S}$ if and only if $\gamma_{1}=\gamma_{1}^{*}$. More precisely, all positive solutions of (5.2) near $\left(S, I, R, \gamma_{1}\right)=\left(S_{0}, 0,0, \gamma_{1}^{*}\right)$ can be parametrized as a smooth curve

$$
\begin{aligned}
\Gamma_{1}=\{ & \left(S, I, R, \gamma_{1}\right)=\left(S_{0}+\left(\phi_{0}+\bar{S}(s)\right) s,\left(\psi_{0}+\bar{I}(s)\right) s,\left(\chi_{0}+\bar{R}(s)\right) s, \gamma_{1}(s)\right) \in X \times \mathbb{R}_{+}: \\
& \left.0<s \leq \epsilon_{0}\right\}
\end{aligned}
$$

for some $\epsilon_{0}>0$. Here, $\left(\bar{S}(s), \bar{I}(s), \bar{R}(s), \gamma_{1}(s)\right)$ is a smooth function of $s$ that satisfies

$$
\left(\bar{S}(0), \bar{I}(0), \bar{R}(0), \gamma_{1}(0)\right)=\left(0,0,0, \gamma_{1}^{*}\right) \text { and } \int_{\Omega} \bar{I}(s) \psi_{0}(x) \mathrm{d} x=0,
$$

$\psi_{0}>0$ is the principal eigenfunction of (5.3), and $\phi_{0}, \chi_{0}$ satisfy

$$
\begin{array}{ll}
-\nabla \cdot\left(d_{S}(x) \nabla \phi_{0}(x)\right)+\mu(x) \phi_{0}(x)=\left[\gamma_{1}^{*}-\frac{\partial F}{\partial I}\left(x, S_{0}, 0\right)\right] \psi_{0}(x)+\delta(x) \chi_{0}(x), & x \in \Omega, \\
-\nabla \cdot\left(d_{R}(x) \nabla \chi_{0}(x)\right)+[\mu(x)+\delta(x)] \chi_{0}(x)=\gamma_{2}(x) \psi_{0}(x) & x \in \Omega, \\
{\left[d_{S}(x) \nabla \phi_{0}(x)\right] \cdot \mathbf{n}=\left[d_{R}(x) \nabla \chi_{0}(x)\right] \cdot \mathbf{n}=0,} & x \in \partial \Omega .
\end{array}
$$

Moreover, $\gamma_{1}^{\prime}(0)$ can be calculated by

$$
\gamma_{1}^{\prime}(0)=\frac{L}{\int_{\Omega} \psi_{0}^{2}(x) \mathrm{d} x}
$$

where

$$
L=\int_{\Omega}\left[\frac{1}{2} \cdot \frac{\partial^{2} f}{\partial I^{2}}\left(x, S_{0}(x), 0\right) \psi_{0}^{3}(x)+\frac{\partial^{2} f}{\partial S \partial I}\left(x, S_{0}(x), 0\right) \phi_{0}(x) \psi_{0}^{2}(x)\right] \mathrm{d} x .
$$

Proof. We begin by making the change of variables $u=\tilde{S}-S_{0}, v=\tilde{I}, w=\tilde{R}$, so the disease-free steady state $(\tilde{S}, \tilde{I}, \tilde{R})=\left(S_{0}(\cdot), 0,0\right)$ is shifted to the origin $(u, v, w)=(0,0,0)$.

Let $Y=L^{p}(\Omega)$. Define a mapping $\mathcal{G}: X \times \mathbb{R}_{+} \rightarrow Y \times Y \times Y$ by

$$
\begin{aligned}
\mathcal{G}\left(u, v, w, \gamma_{1}\right)= & \left(\begin{array}{c}
\nabla \cdot\left(d_{S}(x) \nabla u\right)+f\left(u+S_{0}, v, w, \gamma_{1}\right) \\
d_{I} \Delta v+g\left(u+S_{0}, v, w, \gamma_{1}\right) \\
\nabla \cdot\left(d_{R}(x) \nabla w\right)+h\left(u+S_{0}, v, w, \gamma_{1}\right)
\end{array}\right) \\
= & \left(\begin{array}{c}
\nabla \cdot\left(d_{S}(x) \nabla u\right)+\Lambda(x)-\mu(x)\left(u+S_{0}\right)-F\left(x, u+S_{0}, v\right)+\gamma_{1} v+\delta(x) w \\
d_{I} \Delta v+F\left(x, u+S_{0}, v\right)-\left(\mu+\alpha+\gamma_{2}\right)(x) v-\gamma_{1} v \\
\nabla \cdot\left(d_{R}(x) \nabla w\right)+\gamma_{2}(x) v-\mu(x) w-\delta(x) w
\end{array}\right) .
\end{aligned}
$$


Let $\tilde{f}\left(u, v, w, \gamma_{1}\right)=f\left(u+S_{0}, v, w, \gamma_{1}\right), \tilde{g}\left(u, v, w, \gamma_{1}\right)=g\left(u+S_{0}, v, w, \gamma_{1}\right)$ and $\tilde{h}\left(u, v, w, \gamma_{1}\right)=h(u+$ $\left.S_{0}, v, w, \gamma_{1}\right)$. The Fréchet derivative of $\mathcal{G}$ with respect to $(u, v, w)$ at $\left(u, v, w, \gamma_{1}\right)=\left(0,0,0, \gamma_{1}\right)$ is given by

$$
\begin{aligned}
\mathcal{G}_{(u, v, w)}\left(0,0,0, \gamma_{1}\right)[\phi, \psi, \chi] \\
=\left(\begin{array}{c}
\nabla \cdot\left(d_{S}(x) \nabla \phi\right)+\tilde{f}_{u}\left(0,0,0, \gamma_{1}\right) \phi+\tilde{f}_{v}\left(0,0,0, \gamma_{1}\right) \psi+\tilde{f}_{w}\left(0,0,0, \gamma_{1}\right) \chi \\
d_{I} \Delta \psi+\tilde{g}_{u}\left(0,0,0, \gamma_{1}\right) \phi+\tilde{g}_{v}\left(0,0,0, \gamma_{1}\right) \psi+\tilde{g}_{w}\left(0,0,0, \gamma_{1}\right) \chi \\
\nabla \cdot\left(d_{R}(x) \nabla \chi\right)+\tilde{h}_{u}\left(0,0,0, \gamma_{1}\right) \phi+\tilde{h}_{v}\left(0,0,0, \gamma_{1}\right) \psi+\tilde{h}_{w}\left(0,0,0, \gamma_{1}\right) \chi
\end{array}\right) \\
=\left(\begin{array}{c}
\nabla \cdot\left(d_{S}(x) \nabla \phi\right)-\mu(x) \phi+\left[\gamma_{1}-\frac{\partial F}{\partial I}\left(x, S_{0}, 0\right)\right] \psi+\delta(x) \chi \\
d_{I} \Delta \psi+\left[\frac{\partial F}{\partial I}\left(x, S_{0}, 0\right)-\left(\mu+\alpha+\gamma_{2}\right)(x)-\gamma_{1}\right] \psi \\
\nabla \cdot\left(d_{R}(x) \nabla \chi\right)+\gamma_{2}(x) \psi-[\mu(x)+\delta(x)] \chi
\end{array}\right) .
\end{aligned}
$$

In particular,

$$
\mathcal{G}_{(u, v, w)}\left(0,0,0, \gamma_{1}^{*}\right)[\phi, \psi, \chi]=\left(\begin{array}{c}
\nabla \cdot\left(d_{S}(x) \nabla \phi\right)-\mu(x) \phi-\left[\frac{\partial F}{\partial I}\left(x, S_{0}, 0\right)-\gamma_{1}^{*}\right] \psi+\delta(x) \chi \\
d_{I} \Delta \psi-\gamma_{1}^{*} \psi+H(x) \psi \\
\nabla \cdot\left(d_{R}(x) \nabla \chi\right)+\gamma_{2}(x) \psi-[\mu(x)+\delta(x)] \chi
\end{array}\right) .
$$

We recall that the positive eigenfunction $\psi_{0}$ of (5.3) satisfies

$$
d_{I} \Delta \psi_{0}-\gamma_{1}^{*} \psi_{0}+H(x) \psi_{0}=0 .
$$

If we define

$$
\chi_{0}=-\left[\nabla \cdot\left(d_{R}(x) \nabla\right)-\mu(x)-\delta(x)\right]^{-1} \gamma_{2}(x) \psi_{0}
$$

and

$$
\phi_{0}=\left[\nabla \cdot\left(d_{S}(x) \nabla\right)-\mu(x)\right]^{-1}\left[\left(\frac{\partial F}{\partial I}\left(x, S_{0}, 0\right)-\gamma_{1}^{*}\right) \psi_{0}-\delta(x) \chi_{0}\right],
$$

we can see that $\left(\phi_{0}, \psi_{0}, \chi_{0}\right)$ satisfies

$$
\mathcal{G}_{(u, v, w)}\left(0,0,0, \gamma_{1}^{*}\right)\left[\phi_{0}, \psi_{0}, \chi_{0}\right]=0 .
$$

Hence, $\left(\phi_{0}, \psi_{0}, \chi_{0}\right) \in \operatorname{ker} \mathcal{G}_{(u, v, w)}\left(0,0,0, \gamma_{1}^{*}\right) \backslash\{0\}$, so $\operatorname{ker} \mathcal{G}_{(u, v, w)}\left(0,0,0, \gamma_{1}^{*}\right)$ is non-trivial.

On the other hand, it follows from the definition of $\gamma_{1}^{*}$ that the principal eigenvalue $\lambda^{*}$ of (3.3) equals zero when $\gamma_{1}=\gamma_{1}^{*}$. Since $\lambda^{*}=0$ is a simple eigenvalue, the eigenfunction $\psi_{0}$ is unique up to constant multiples. Moreover, $\chi_{0}$ and $\phi_{0}$ are uniquely determined by (5.10) and (5.11) for a fixed $\psi_{0}$. This implies that $\operatorname{ker} \mathcal{G}_{(u, v, w)}\left(0,0,0, \gamma_{1}^{*}\right)=\operatorname{span}\left\{\left(\phi_{0}, \psi_{0}, \chi_{0}\right)\right\}$.

It is easy to see that $\left(h_{1}, h_{2}, h_{3}\right) \in \operatorname{range} \mathcal{G}_{(u, v, w)}\left(0,0,0, \gamma_{1}^{*}\right)$ if and only if there exists $(\phi, \psi, \chi) \in X$ such that

$$
\begin{aligned}
& h_{1}=\nabla \cdot\left(d_{S}(x) \nabla \phi\right)-\mu(x) \phi-\left[\frac{\partial F}{\partial I}\left(x, S_{0}, 0\right)-\gamma_{1}^{*}\right] \psi+\delta(x) \chi, \\
& h_{2}=d_{I} \Delta \psi-\gamma_{1}^{*} \psi+H(x) \psi \\
& h_{3}=\nabla \cdot\left(d_{R}(x) \nabla \chi\right)+\gamma_{2}(x) \psi-[\mu(x)+\delta(x)] \chi .
\end{aligned}
$$

It follows from the Fredholm alternative theorem that the second equation of the above system is solvable if and only if $\int_{\Omega} h_{2}(x) \psi_{0}(x) \mathrm{d} x=0$. Then, for the obtained solution $\psi$, the third equation of (5.12) has a unique solution

$$
\chi=\left[\nabla \cdot\left(d_{R}(x) \nabla\right)-\mu(x)-\delta(x)\right]^{-1}\left[h_{3}-\gamma_{2}(x) \psi\right] .
$$

Then, for such $\psi$ and $\chi$, the first equation of (5.12) has a unique solution

$$
\phi=\left[\nabla \cdot\left(d_{S}(x) \nabla\right)-\mu(x)\right]^{-1}\left[h_{1}+\left[\frac{\partial F}{\partial I}\left(x, S_{0}, 0\right)-\gamma_{1}^{*}\right] \psi-\delta(x) \chi\right] .
$$


From this, it follows that the range of $\mathcal{G}_{(u, v, w)}\left(0,0,0, \gamma_{1}^{*}\right)$ is given by

$$
\text { range } \mathcal{G}_{(u, v, w)}\left(0,0,0, \gamma_{1}^{*}\right)=\left\{\left(h_{1}, h_{2}, h_{3}\right) \in Y^{3}: \int_{\Omega} h_{2}(x) \psi_{0}(x) \mathrm{d} x=0\right\} .
$$

The above equation defines a constraint on the variable $h_{2}$. Since there are no constraints on $h_{1}$ or $h_{3}$, the solution to system (5.12) has two degrees of freedom. This shows that dim range $\mathcal{G}_{(u, v, w)}\left(0,0,0, \gamma_{1}^{*}\right)=2$ and thus, codim range $\mathcal{G}_{(u, v, w)}\left(0,0,0, \gamma_{1}^{*}\right)=1$.

Furthermore, we can calculate

$$
\begin{aligned}
\mathcal{G}_{(u, v, w), \gamma_{1}}\left(0,0,0, \gamma_{1}^{*}\right)\left[\phi_{0}, \psi_{0}, \chi_{0}\right] \\
=\left(\begin{array}{c}
\tilde{f}_{u, \gamma_{1}}\left(0,0,0, \gamma_{1}^{*}\right) \phi_{0}+\tilde{f}_{v, \gamma_{1}}\left(0,0,0, \gamma_{1}^{*}\right) \psi_{0}+\tilde{f}_{w, \gamma_{1}}\left(0,0,0, \gamma_{1}^{*}\right) \chi_{0} \\
\tilde{g}_{u, \gamma_{1}}\left(0,0,0, \gamma_{1}^{*}\right) \phi_{0}+\tilde{g}_{v, \gamma_{1}}\left(0,0,0, \gamma_{1}^{*}\right) \psi_{0}+\tilde{g}_{w, \gamma_{1}}\left(0,0,0, \gamma_{1}^{*}\right) \chi_{0} \\
\tilde{h}_{u, \gamma_{1}}\left(0,0,0, \gamma_{1}^{*}\right) \phi_{0}+\tilde{h}_{v, \gamma_{1}}\left(0,0,0, \gamma_{1}^{*}\right) \psi_{0}+\tilde{h}_{w, \gamma_{1}}\left(0,0,0, \gamma_{1}^{*}\right) \chi_{0}
\end{array}\right) \\
=\left(\begin{array}{c}
\psi_{0} \\
-\psi_{0} \\
0
\end{array}\right),
\end{aligned}
$$

where $\mathcal{G}_{(u, v, w), \gamma_{1}}$ denotes the second partial Fréchet derivative of $\mathcal{G}$ with respect to the variables $(u, v, w)$ and $\gamma_{1}$. Similarly, we denote by $\tilde{f}_{u, \gamma_{1}}$ the second partial derivative of $\tilde{f}$ with respect to $u$ and $\gamma_{1}$, and so on.

Since $\int_{\Omega}\left[-\psi_{0}(x)\right] \psi_{0}(x) \mathrm{d} x=-\int_{\Omega}\left[\psi_{0}(x)\right]^{2} \mathrm{~d} x<0$, we obtain

$$
\mathcal{G}_{(u, v, w), \gamma_{1}}\left(0,0,0, \gamma_{1}^{*}\right)\left[\phi_{0}, \psi_{0}, \chi_{0}\right] \notin \text { range } \mathcal{G}_{(u, v, w)}\left(0,0,0, \gamma_{1}^{*}\right)
$$

This allows us to apply the theorem of bifurcation from a simple eigenvalue [35] to conclude that the set of positive solutions to (5.2) near $\left(S, I, R, \gamma_{1}\right)=\left(S_{0}(\cdot), 0,0, \gamma_{1}^{*}\right)$ is a curve of the form (5.6). We also note that the possibility of other bifurcation points different from $\gamma_{1}=\gamma_{1}^{*}$ is excluded by virtue of the Krein-Rutman theorem. Furthermore, according to the direction of bifurcation formula by Shi [37], we have

$$
\gamma_{1}^{\prime}(0)=-\frac{\left\langle l, \mathcal{G}_{(u, v, w),(u, v, w)}\left(0,0,0, \gamma_{1}^{*}\right)\left[\phi_{0}, \psi_{0}, \chi_{0}\right]^{2}\right\rangle}{2\left\langle l, \mathcal{G}_{(u, v, w), \gamma_{1}}\left(0,0,0, \gamma_{1}^{*}\right)\left[\phi_{0}, \psi_{0}, \chi_{0}\right]\right\rangle},
$$

where the linear functional $l: Y^{2} \rightarrow \mathbb{R}$ is defined as

$$
\left\langle l,\left[h_{1}, h_{2}, h_{3}\right]\right\rangle=\int_{\Omega} h_{2}(x) \psi_{0}(x) \mathrm{d} x,
$$

while $\mathcal{G}_{(u, v, w),(u, v, w)}$ and $\mathcal{G}_{(u, v, w), \gamma_{1}}$ denote the second partial Fréchet derivatives of $\mathcal{G}$.

By direct calculation, we can see that the second component of

$$
\mathcal{G}_{(u, v, w),(u, v, w)}\left(0,0,0, \gamma_{1}^{*}\right)\left[\phi_{0}, \psi_{0}, \chi_{0}\right]^{2}
$$

takes the form

$$
\begin{aligned}
G_{0}(x):= & \tilde{g}_{u u}\left(0,0,0, \gamma_{1}^{*}\right) \phi_{0}^{2}+\tilde{g}_{v v}\left(0,0,0, \gamma_{1}^{*}\right) \psi_{0}^{2}+\tilde{g}_{w w}\left(0,0,0, \gamma_{1}^{*}\right) \chi_{0}^{2} \\
& +2 \tilde{g}_{u v}\left(0,0,0, \gamma_{1}^{*}\right) \phi_{0} \psi_{0}+2 \tilde{g}_{v w}\left(0,0,0, \gamma_{1}^{*}\right) \psi_{0} \chi_{0}+2 \tilde{g}_{w u}\left(0,0,0, \gamma_{1}^{*}\right) \chi_{0} \phi_{0} \\
= & \frac{\partial^{2} f}{\partial I^{2}}\left(x, S_{0}(x), 0\right) \psi_{0}^{2}+2 \frac{\partial^{2} f}{\partial S \partial I}\left(x, S_{0}(x), 0\right) \phi_{0} \psi_{0} .
\end{aligned}
$$


Thus,

$$
\gamma_{1}^{\prime}(0)=-\frac{\int_{\Omega} G_{0}(x) \psi_{0}(x) \mathrm{d} x}{2\left[-\int_{\Omega} \psi_{0}^{2}(x) \mathrm{d} x\right]}=\frac{L}{\int_{\Omega} \psi_{0}^{2}(x) \mathrm{d} x},
$$

where $L$ is given by (5.9). This completes the proof.

Next, we use the global bifurcation theory to give an extension of the local bifurcation branch (5.6). We define the set of positive solutions of (5.2) as

$$
\Sigma=\left\{\left(S, I, R, \gamma_{1}\right) \in X \times \mathbb{R}_{+}:\left(S, I, R, \gamma_{1}\right) \text { is a solution of (5.2)with } S, I, R, \gamma_{1}>0\right\} .
$$

We can thus obtain the following result.

Theorem 5.3. The set of positive solutions of (5.2) with bifurcation parameter $\gamma_{1}$ forms a continuum $\Sigma_{1} \subset X \times \mathbb{R}_{+}$that bifurcates from the disease-free steady state $\left(S_{0}, 0,0\right)$ when $\mathcal{R}_{0}=1$.

Moreover, $\Sigma_{1}$ is a connected component of $\bar{\Sigma}$ containing the curve $\Gamma_{1}$. The projection $\operatorname{proj}_{\gamma_{1}} \Sigma_{1}$ of $\Sigma_{1}$ into the $\gamma_{1}$-axis satisfies

$$
\operatorname{proj}_{\gamma_{1}} \Sigma_{1}=\left[0, \gamma_{1}^{*}\right]
$$

and $\Sigma_{1}$ contains a point of the form $(\hat{S}, \hat{I}, \hat{R}, 0)$, where $(\hat{S}, \hat{I}, \hat{R})$ is a positive solution of $(5.2)$ with $\gamma_{1}=0$. Proof. For the local bifurcation branch obtained in Theorem 5.2, let $\Sigma_{1}$ be any maximal extension in $X \times \mathbb{R}_{+}$as a connected set of solutions of (5.2). From the remarks in [38], we can verify that all conditions in [38, Theorem 4.4] are satisfied. Therefore, $\Sigma_{1}$ must satisfy one of the following:

(i) it is not compact; or

(ii) it contains a point $\left(S_{0}, 0,0, \hat{\gamma}_{1}\right)$ with $\hat{\gamma}_{1} \neq \gamma_{1}^{*}$; or

(iii) it contains a point $\left(S_{0}+U, V, W, \gamma_{1}\right)$ where $(U, V, W) \neq 0$ and $(U, V, W)$ is in the complement of $\operatorname{span}\left\{\phi_{0}, \psi_{0}, \chi_{0}\right\}$.

Since the eigenvalue of (5.3) with positive eigenfunction is unique, it is clear that (ii) cannot occur.

Now, by Theorem 3.3(i), system (5.2) has no positive steady state solutions for $\mathcal{R}_{0}<1$, that is, when $\gamma_{1}>\gamma_{1}^{*}$. This implies that $\operatorname{proj}_{\gamma_{1}} \Sigma_{1} \subset\left[0, \gamma_{1}^{*}\right]$. On the other hand, due to Theorem 4.1, there exists a positive steady state for all $\gamma_{1}<\gamma_{1}^{*}$ (which corresponds to $\mathcal{R}_{0}>1$ ). Therefore, we can conclude that $\operatorname{proj}_{\gamma_{1}} \Sigma_{1}=\left[0, \gamma_{1}^{*}\right]$. This result, together with the boundedness of solutions, shows that $\Sigma_{1}$ must be compact.

The above discussion implies that case (iii) holds. Then, by a standard argument with the global bifurcation theorem, we can find a certain $\left(\hat{S}, \hat{I}, \hat{R}, \hat{\gamma}_{1}\right) \in \Sigma_{1}$ such that $\left(\hat{S}, \hat{I}, \hat{R}, \hat{\gamma}_{1}\right)$ is in the boundary of the set of positive solutions $\Sigma$. Since there are no equilibrium states with $\hat{S}=0, \hat{I}=0$ or $\hat{R}=0$ other than the disease-free steady state, the only possibility is $\hat{\gamma}_{1}=0$ and $\hat{S}, \hat{I}, \hat{R}>0$. Hence, $(\hat{S}, \hat{I}, \hat{R})$ is a positive solution of system (5.2) with $\gamma_{1}=0$, which completes the proof.

\section{Numerical simulations}

In this section, we will perform some numerical simulations for system (1.2) to investigate the dynamics of the solutions as some of the parameters are varied. For simplicity, we consider the one-dimensional domain $\Omega=(0,20) \subset \mathbb{R}$. We will use the parameter values

$$
\Lambda=10, \quad \mu=0.001, \quad \alpha=0.01, \quad \gamma_{1}=0.02, \quad \gamma_{2}=0.07, \quad \delta=0.02 .
$$

Furthermore, we consider the saturated incidence function

$$
F(x, S, I)=\frac{\beta S I}{1+a_{1}(x) I},
$$



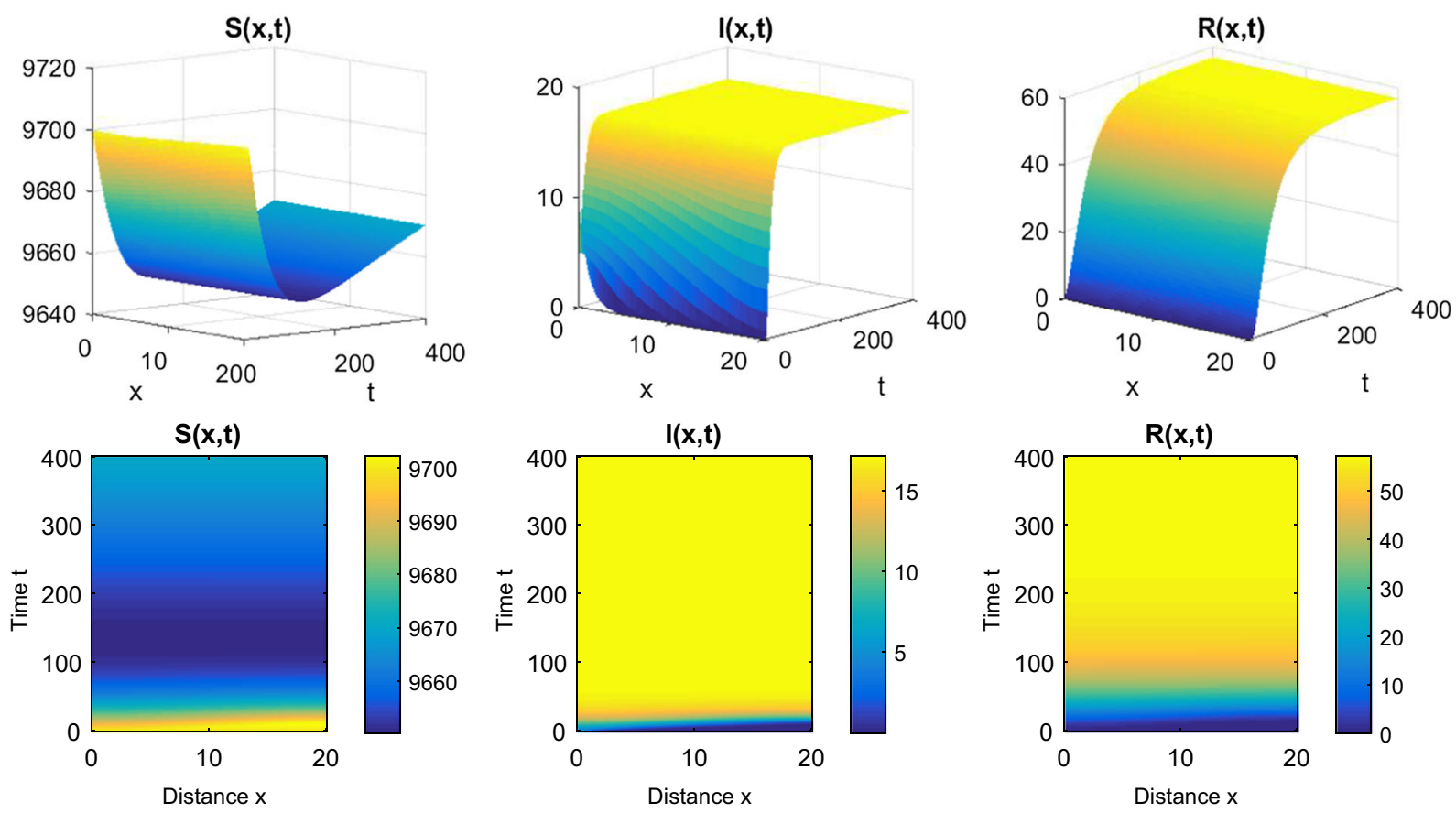

FIG. 1. Time variation of $S(x, t), I(x, t)$ and $R(x, t)$ for system $(1.2)$ with the parameters given in $(6.1), d_{S}=d_{I}=d_{R}=1$ and $a_{1}(x) \equiv 0.5$

where $\beta=0.0001$ and the parameter $a_{1}$ is a positive function of the spatial variable. This allows us to study the case when the incidence function saturates more rapidly in some locations, due to the different measures taken by the population to eradicate the epidemic outbreak. It is readily shown that this form of incidence function satisfies (A1)-(A4).

Throughout this section, we will use the initial conditions

$$
S_{0}(x)=9700, \quad I_{0}(x)=10 \exp (-x), \quad R_{0}(x)=0 \quad \text { for } x \in[0,20]
$$

\subsection{Effect of heterogeneous saturation rate}

We will first study the effect that heterogeneity in the saturation parameter has on the model dynamics. We define the function $a_{1}: \Omega \rightarrow \mathbb{R}_{+}$by $a_{1}(x)=0.5[1+k \sin (2 \pi x / 20)]$, where $k \in[0,1]$ is a number that represents the magnitude of spatial heterogeneity. The case $k=0$ corresponds to $a_{1}(x) \equiv 0.5$.

Now, we fix the values of the diffusion rates as $d_{S}=d_{I}=d_{R}=1$ and plot in Fig. 1 the solutions to system (1.2) when $k=0$, that is, when the incidence rate $F(x, S, I)=\beta S I /\left(1+a_{1} I\right)$ is independent of $x$. In this case, the densities of susceptible, infected and recovered populations converge to positive constants.

If we increase the heterogeneity by choosing $k=0.95$ while keeping all other parameter values as before, we obtain the solutions plotted in Fig. 2. Here, the values of $S(x, t), I(x, t)$ and $R(x, t)$ tend to a non-homogeneous endemic equilibrium as $t \rightarrow \infty$.

Lastly, we plot the spatial distribution of susceptible, infected and recovered populations at $t=1000$ for $k=0,0.5,0.8,0.95$ in Fig. 3. We can see that greater heterogeneity in the saturation parameter results in higher values of infected and recovered populations. 

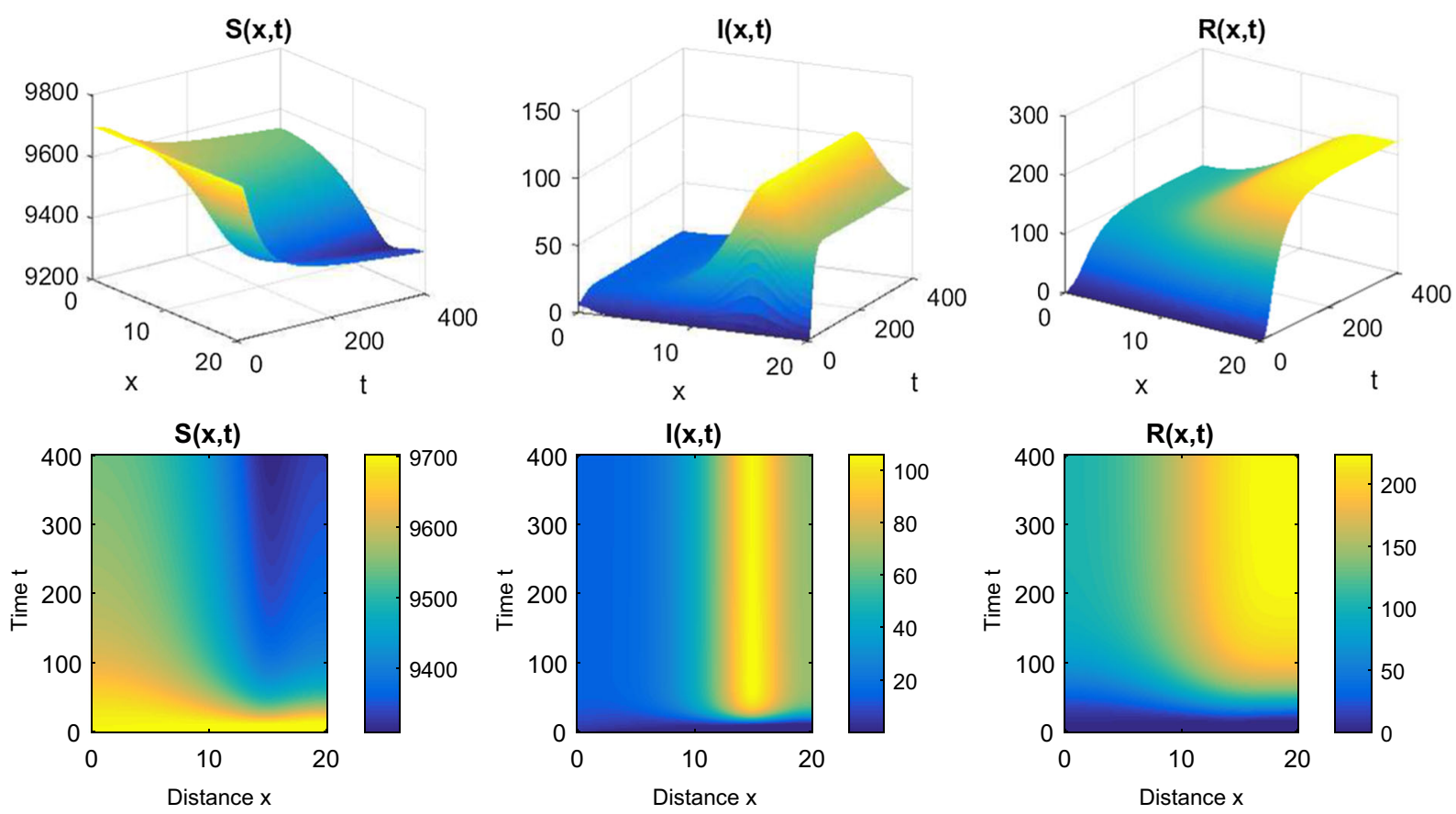

Fig. 2. Time variation of $S(x, t), I(x, t)$ and $R(x, t)$ for system $(1.2)$ with the parameters given in $(6.1), d_{S}=d_{I}=d_{R}=1$ and $a_{1}(x)=0.5[1+0.95 \sin (2 \pi x / 20)]$

\subsection{Effect of varying the diffusion rates}

We will consider now the effects of using different diffusion rates for system (1.2) while keeping all other parameter values fixed. Here, we assume that $a_{1}(x)=0.5[1+0.95 \sin (2 \pi x / 20)]$ and other parameters are taken as in (6.1).

Suppose first that the diffusion rate of infected population is fixed as $d_{I}=1$, while the diffusion rates of susceptible and recovered populations are both equal to some constant $D$. Figure 4 a depicts the distribution of $S, I$ and $R$ at $t=1000$ for several values of $D$. The figure shows that reducing the value of the diffusion rates $d_{S}=d_{R}=D$ contributes to reducing the infected population levels.

Next, we make some simulations assuming that the diffusion rates $d_{S}, d_{I}$ and $d_{R}$ are all equal to $D$. In this case, the spatial distribution of solutions is shown in Fig. 4b. Our simulations show that reducing the diffusion rate in all three subpopulations results in greater heterogeneity for the distribution of the infected; however, the density of infected population actually increases at certain locations for lower values of $D$. Hence, restricting the motility for all individuals does not necessarily reduce the total number of infections. 

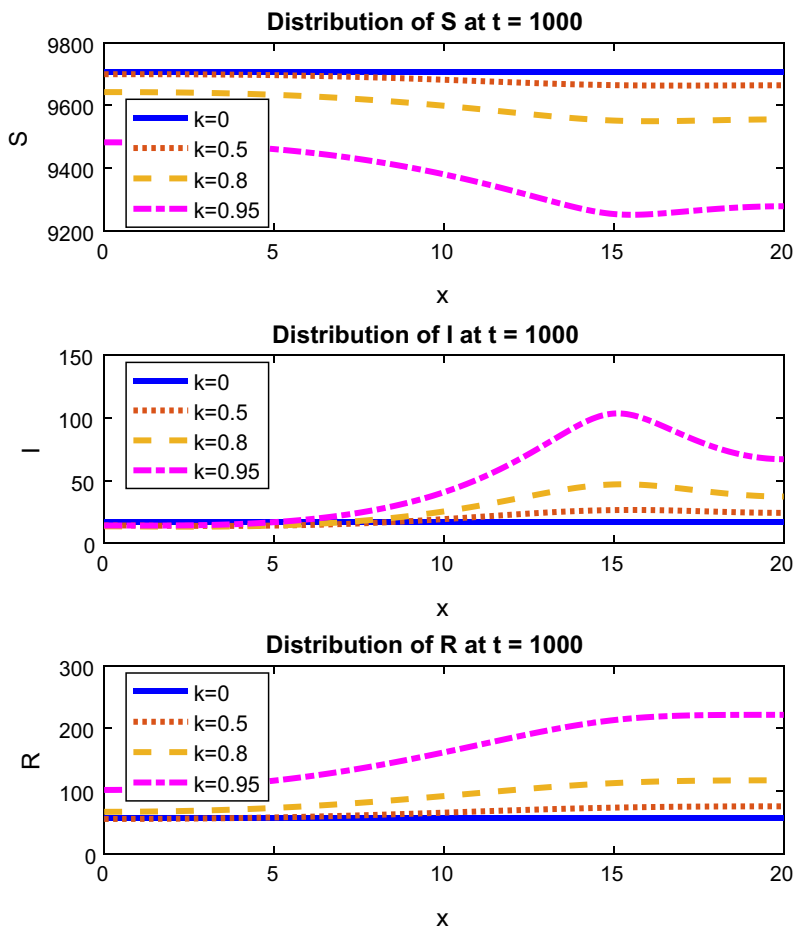

FIG. 3. Spatial distribution of $S(x, t), I(x, t)$ and $R(x, t)$ at $t=1000$ for system (1.2) with $d_{S}=d_{I}=d_{R}=1$ and $a_{1}(x)=0.5[1+k \sin (2 \pi x / 20)]$ for several values of $k$. The other parameter values are given in $(6.1)$

\subsection{Simulations of bifurcation when varying the transfer rate from infected to susceptible}

We will now simulate the bifurcation dynamics of our model when the parameter $\gamma_{1}$ is varied. Here, we fix the parameter values

$$
\begin{aligned}
& \Lambda=10, \quad \mu=0.001, \quad \alpha=0.01, \quad \gamma_{2}=0.07, \quad \delta=0.02, \quad d_{S}=d_{I}=d_{R}=1, \\
& F(x, S, I)=\frac{\beta S I}{1+a_{1}(x) I}, \quad a_{1}(x)=0.5\left[1+0.95 \sin \left(\frac{2 \pi x}{20}\right)\right]
\end{aligned}
$$

and let $\gamma_{1}$ vary from 0 to 0.8 .

Using the same initial conditions as before, we obtain the distribution of susceptible, infected and recovered individuals shown in Fig. 5 for $t=4000$. We can see that for $\gamma_{1} \in[0,0.6]$, the solution tends to a positive endemic steady state, which corresponds to the case when $\mathcal{R}_{0}>1$. As we increase the value of $\gamma_{1}$, the infected population decreases. The basic reproduction number crosses unity at a certain value $\gamma_{1}^{*} \in(0.6,0.8)$, and there is no endemic equilibrium for $\gamma_{1}>\gamma_{1}^{*}$. Figure 5 shows that the solutions of the model tend to the disease-free steady state $\left(E_{0}=\left(10^{4}, 0,0\right)\right)$ when $\gamma_{1}=0.8$.

\section{Conclusions}

We proposed a diffusive SIRS epidemic model that generalizes several previously studied models in the literature, such as $[9-11,18]$. In particular, we extended the results obtained by Yang et al. in [18]by 

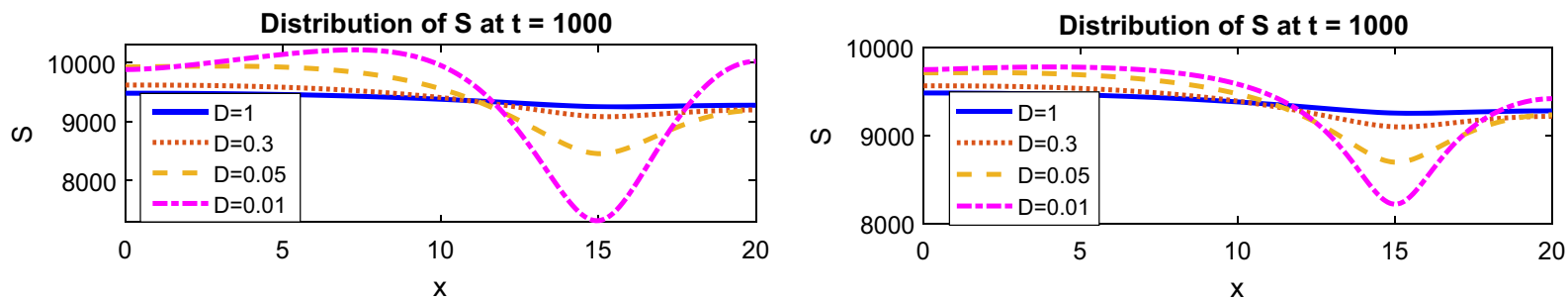

Distribution of $\mathrm{I}$ at $\mathrm{t}=1000$
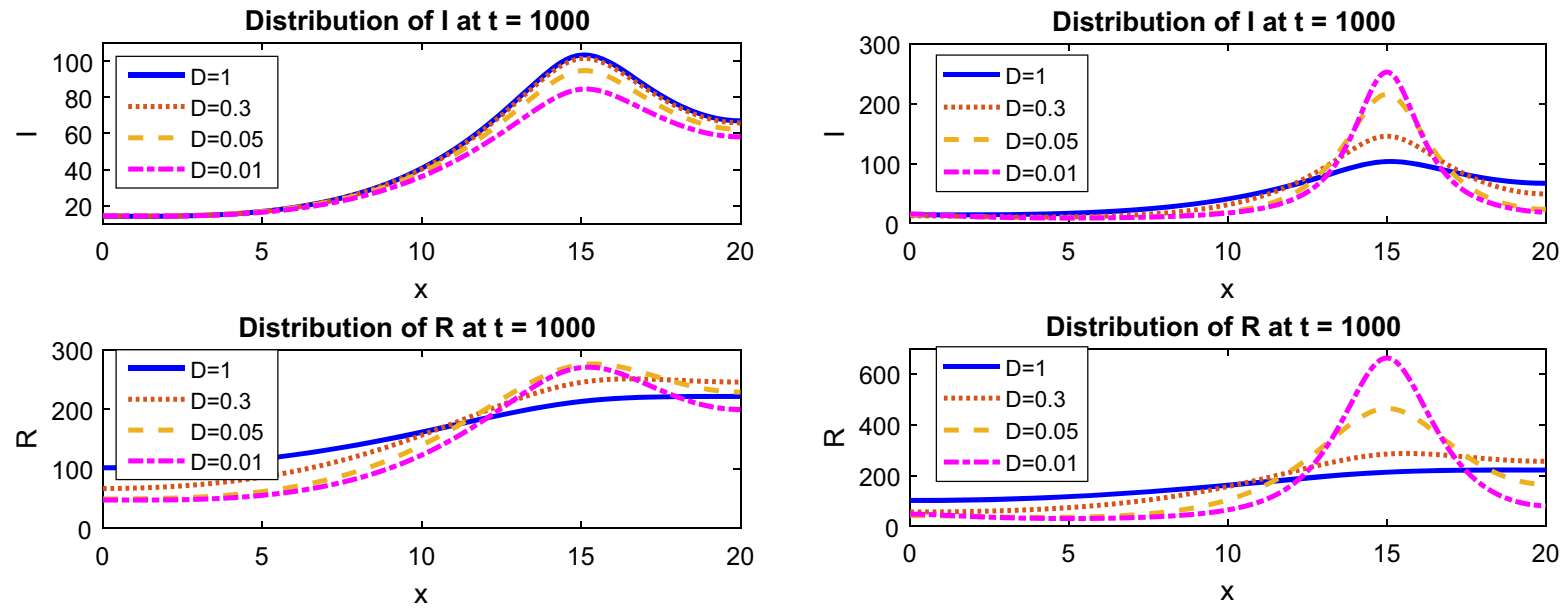

(A)

(B)

FIG. 4. Spatial distribution of $S(x, t), I(x, t)$ and $R(x, t)$ at $t=1000$ for system (1.2) with the parameters given in (6.1), $a_{1}(x)=0.5[1+0.95 \sin (2 \pi x / 20)]$ and several values for the diffusion rates. a $d_{S}=D, d_{I}=1, d_{R}=D$, where $D$ varies from 1 to 0.01 . $\mathbf{b} d_{S}=d_{I}=d_{R}=D$, where $D$ varies from 1 to 0.01

considering a model with three different diffusion rates, $d_{S}(x), d_{I}(x)$ and $d_{R}(x)$, which may vary depending on the location of individuals. Moreover, our model assumes that the incidence function takes the general form $F(x, S, I)$, which includes many different types of nonlinear or non-monotone functions and allows for spatial heterogeneity.

We established the threshold dynamics for model (1.2) with respect to the basic reproduction number: the disease-free steady state is globally asymptotically stable when $\mathcal{R}_{0}<1$, while the disease persists and an endemic equilibrium appears when $\mathcal{R}_{0}>1$.

Furthermore, we performed a bifurcation analysis for model (1.2) in the case when the diffusion coefficient of infected population and the rate of transfer from infected to susceptible classes are constant. We used the theory by Crandall and Rabinowitz $[35,37,38]$ to determine the type of bifurcation that occurs when the basic reproduction number crosses unity. From Theorems 5.2 and 5.3, we can infer that the model always undergoes a forward bifurcation at $\mathcal{R}_{0}=1$, and the existence of endemic equilibria is completely ruled out when $\mathcal{R}_{0}$ is less than one.

We also carried out some simulations to illustrate the effects of heterogeneity on the dynamics of model (1.2). We used, as an example, the incidence rate of the form $F(x, S, I)=\beta S I /\left[1+a_{1}(x) I\right]$, which does not meet the assumptions considered in [18] but is included by our general model. Our examples show that a larger variation in the incidence rate may increase the number of infections in the population. 

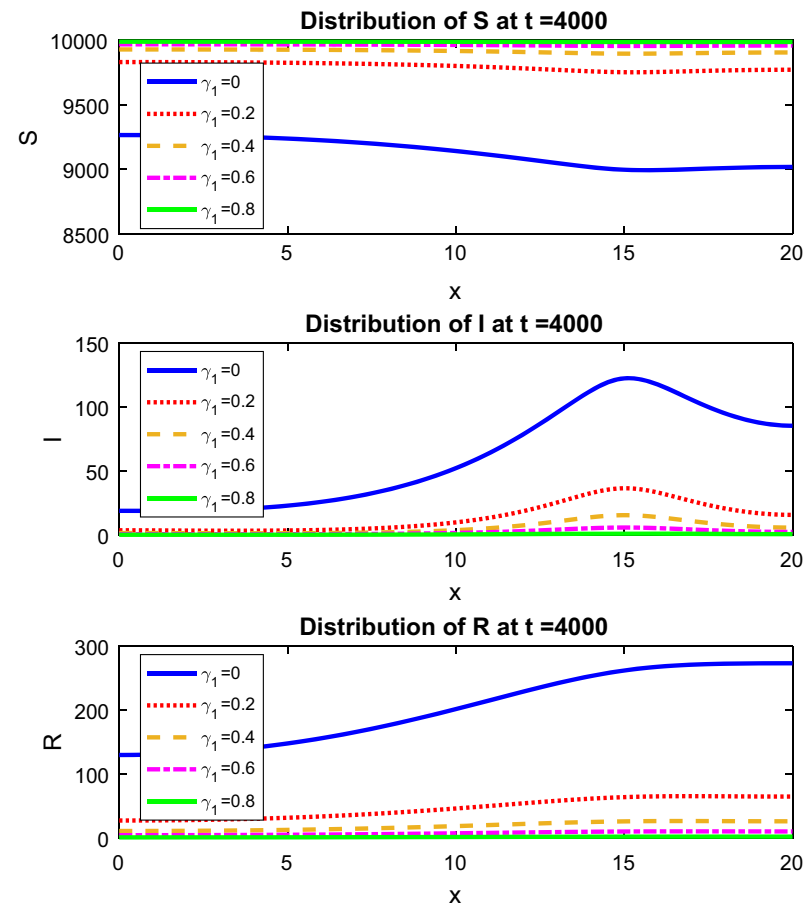

FIG. 5. Spatial distribution of $S(x, t), I(x, t)$ and $R(x, t)$ at $t=4000$ for system (1.2) with the parameter values given in (6.3) and several values for $\gamma_{1}$

\section{Acknowledgements}

This work was supported by Sistema Nacional de Investigadores (15284) and Conacyt-Becas [Consejo Nacional de Ciencia y Tecnología (Grant No. 777140)].

Publisher's Note Springer Nature remains neutral with regard to jurisdictional claims in published maps and institutional affiliations.

\section{References}

[1] Anderson, R.M., May, R.M.: Population biology of infectious diseases: part I. Nature 280(5721), 361-367 (1979)

[2] Kumar, A., Nilam: Stability of a time delayed SIR epidemic model along with nonlinear incidence rate and Holling type-II treatment rate. Int. J. Comput. Methods 15(6), 1850055 (2018)

[3] Wei, C., Chen, L.: A delayed epidemic model with pulse vaccination. Discrete Dyn. Nat. Soc. 2008, 746951 (2008)

[4] Dubey, B., Dubey, P., Dubey, U.S.: Dynamics of an SIR model with nonlinear incidence and treatment rate. Appl. Appl. Math. 10(2), 718-737 (2015)

[5] Upadhyay, R.K., et al.: Dynamics of an SEIR epidemic model with nonlinear incidence and treatment rates. Nonlinear Dyn. 96(4), 2351-2368 (2019)

[6] Cui, J., Sun, Y., Zhu, H.: The impact of media on the control of infectious diseases. J. Dyn. Differ. Equ. 20(1), 31-53 (2008)

[7] Agrawal, A., Tenguria, A., Modi, G.: Stability analysis of an SIR epidemic model with specific nonliner incidence rate. Math. Theory Model. 6(1), 45-51 (2016)

[8] Zhou, Y., Xiao, D., Li, Y.: Bifurcations of an epidemic model with non-monotonic incidence rate of saturated mass action. Chaos Solitons Fractals 32(5), 1903-1915 (2007) 
[9] Li, T., et al.: Threshold dynamics of an SIRS model with nonlinear incidence rate and transfer from infectious to susceptible. Appl. Math. Lett. 70, 52-57 (2017)

[10] Bai, Y., Mu, X.: Global asymptotic stability of a generalized SIRS epidemic model with transfer from infectious to susceptible. J. Appl. Anal. Comput. 8(2), 402-412 (2018)

[11] Avila-Vales, E.J., Cervantes-Pérez, A.G.: Global stability for SIRS epidemic models with general incidence rate and transfer from infectious to susceptible. Bol. Soc. Mat. Mex. 25(3), 637-658 (2019)

[12] Cai, Y., et al.: Spatiotemporal transmission dynamics for influenza disease in a heterogenous environment. Nonlinear Anal. Real World Appl. 46, 178-194 (2019)

[13] Wang, J., Xie, F., Kuniya, T.: Analysis of a reaction-diffusion cholera epidemic model in a spatially heterogeneous environment. Commun. Nonlinear Sci. Numer. Simul. 80, 104951 (2020)

[14] Zhu, D., Ren, J., Zhu, H.: Spatial-temporal basic reproduction number and dynamics for a dengue disease diffusion model. Math. Methods Appl. Sci. 41(14), 5388-5403 (2018)

[15] Yang, J., Xu, R., Li, J.: Threshold dynamics of an age-space structured brucellosis disease model with Neumann boundary condition. Nonlinear Anal. Real World Appl. 50, 192-217 (2019)

[16] Viguerie, A., et al.: Diffusion-reaction compartmental models formulated in a continuum mechanics framework: application to COVID-19, mathematical analysis, and numerical study. Comput. Mech. 66(5), 1131-1152 (2020)

[17] Yang, Y., Zhou, J., Hsu, C.-H.: Threshold dynamics of a diffusive SIRI model with nonlinear incidence rate. J. Math. Anal. Appl. 478(2), 874-896 (2019)

[18] Yang, Y., et al.: Dynamical analysis of a diffusive SIRS model with general incidence rate. Discrete Contin. Dyn. Syst. Ser. B 25(7), 2433-2451 (2020)

[19] Smith, H.L.: Monotone Dynamical Systems: An Introduction to the Theory of Competitive and Cooperative Systems. American Mathematical Society, Providence (1995)

[20] Ren, X., et al.: A reaction-diffusion within-host HIV model with cell-to-cell transmission. J. Math. Biol. 76(7), 1831-1872 (2018)

[21] Luo, Y., et al.: Analysis of a diffusive virus infection model with humoral immunity, cell-to-cell transmission and nonlinear incidence. Physica A 535, 122415 (2019)

[22] Martin, R.H., Smith, H.L.: Abstract functional-differential equations and reaction-diffusion systems. Trans. Am. Math. Soc. 321(1), 1-44 (1990)

[23] Groeger, J.: Divergence theorems and the supersphere. J. Geom. Phys. 77, 13-29 (2014)

[24] Guenther, R.B., Lee, J.W.: Partial Differential Equations of Mathematical Physics and Integral Equations. Dover, New York (1996)

[25] Wang, M.: Nonlinear Elliptic Equations. Science Press, Beijing (2010)

[26] Wu, J.: Theory and Applications of Partial Functional Differential Equations. Springer, New York (1996)

[27] Hale, J.K.: Asymptotic Behavior of Dissipative Systems. American Mathematical Society, Providence (1988)

[28] Diekmann, O., Heesterbeek, J.A.P., Metz, J.A.J.: On the definition and the computation of the basic reproduction ratio R0 in models for infectious diseases in heterogeneous populations. J. Math. Biol. 28(4), 365-382 (1990)

[29] van den Driessche, P., Watmough, J.: Reproduction numbers and sub-threshold endemic equilibria for compartmental models of disease transmission. Math. Biosci. 180(1-2), 29-48 (2002)

[30] Wang, W., Zhao, X.-Q.: A nonlocal and time-delayed reaction-diffusion model of dengue transmission. SIAM J. Appl. Math. 71(1), 147-168 (2011)

[31] Wang, W., Zhao, X.-Q.: Basic reproduction numbers for reaction-diffusion epidemic models. SIAM J. Appl. Dyn. Syst. 11(4), 1652-1673 (2012)

[32] Hess, P.: Periodic-Parabolic Boundary Value Problems and Positivity. Longman, Harlow (1991)

[33] Smith, H.L., Zhao, X.-Q.: Robust persistence for semidynamical systems. Nonlinear Anal. Theory Methods Appl. 47(9), 6169-6179 (2001)

[34] Magal, P., Zhao, X.-Q.: Global attractors and steady states for uniformly persistent dynamical systems. SIAM J. Math. Anal. 37(1), 251-275 (2005)

[35] Crandall, M.G., Rabinowitz, P.H.: Bifurcation from simple eigenvalues. J. Funct. Anal. 8(2), 321-340 (1971)

[36] Ni, W.-M.: The Mathematics of Diffusion. Society for Industrial and Applied Mathematics, Philadelphia (2011)

[37] Shi, J.: Persistence and bifurcation of degenerate solutions. J. Funct. Anal. 169(2), 494-531 (1999)

[38] Shi, J., Wang, X.: On global bifurcation for quasilinear elliptic systems on bounded domains. J. Differ. Equ. 246(7), 2788-2812 (2009) 
Eric Avila-Vales and Ángel G. C. Pérez

Facultad de Matemáticas

Universidad Autónoma de Yucatán

Anillo Periférico Norte, Tablaje Catastral 13615

C.P. 97119 Mérida

Mexico

e-mail: angel.cervantes@correo.uady.mx

Eric Avila-Vales

e-mail: avila@correo.uady.mx

(Received: January 17, 2021; revised: July 9, 2021; accepted: October 17, 2021) 